TRANSACTIONS OF THE

AMERICAN MATHEMATICAL SOCIETY

Volume 360, Number 4, April 2008, Pages 2089-2130

S 0002-9947(07)04363-2

Article electronically published on November 28, 2007

\title{
CONFORMAL ENERGY, CONFORMAL LAPLACIAN, AND ENERGY MEASURES ON THE SIERPINSKI GASKET
}

\author{
JONAS AZZAM, MICHAEL A. HALL, AND ROBERT S. STRICHARTZ
}

\begin{abstract}
On the Sierpinski Gasket (SG) and related fractals, we define a notion of conformal energy $\mathcal{E}_{\varphi}$ and conformal Laplacian $\Delta_{\varphi}$ for a given conformal factor $\varphi$, based on the corresponding notions in Riemannian geometry in dimension $n \neq 2$. We derive a differential equation that describes the dependence of the effective resistances of $\mathcal{E}_{\varphi}$ on $\varphi$. We show that the spectrum of $\Delta_{\varphi}$ (Dirichlet or Neumann) has similar asymptotics compared to the spectrum of the standard Laplacian, and also has similar spectral gaps (provided the function $\varphi$ does not vary too much). We illustrate these results with numerical approximations. We give a linear extension algorithm to compute the energy measures of harmonic functions (with respect to the standard energy), and as an application we show how to compute the $L^{p}$ dimensions of these measures for integer values of $p \geq 2$. We derive analogous linear extension algorithms for energy measures on related fractals.
\end{abstract}

\section{INTRODUCTION}

In Riemannian geometry, a conformal change of metric induces a well-defined change in curvature, energy, and Laplacian. For example, when the dimension $n=2$, the energy is unchanged. In all other dimensions, the new energy uniquely determines the conformal factor (it is simply the integral of the gradient squared with respect to a measure that is the old Riemannian measure multiplied by a power of the conformal factor).

In fractal analysis we have, as yet, no analogue of Riemannian metric or curvature, but we do have energy and Laplacian (see [18] for a discussion of these matters). So we will construct an analogue of "conformal change" by considering a change in energy obtained by multiplying all energy measures by a conformal factor $\varphi(x)$. We neeed $\varphi$ to be positive and measurable, and to avoid technicalities we will assume

$$
0<c_{1} \leq \varphi(x) \leq c_{2}
$$

Received by the editors March 27, 2006.

2000 Mathematics Subject Classification. Primary 28A80.

The first and second authors were supported by the National Science Foundation through the Research Experiences for Undergraduates (REU) program at Cornell.

The third author was supported in part by the National Science Foundation, grant DMS0140194 . 
although in the future it might be desirable to drop these conditions. We definitely do not want to assume that $\varphi$ is continuous, as our basic examples will be step functions.

Suppose, to be specific, we have a self-similar energy $\mathcal{E}$ on a p.c.f, self-similar fractal $K([8],[22])$. For the most part we will deal with the standard energy on the Sierpinski Gasket $S G$. Recall that $S G$ is the unique nonempty compact set in $\mathbb{R}^{2}$ satisfying

$$
K=\bigcup_{i} F_{i} K
$$

where $F_{i}(x)=\frac{1}{2} x+\frac{1}{2} q_{i}$ and $\left\{q_{0}, q_{1}, q_{2}\right\}=V_{0}$ are the vertices of a triangle (we call $V_{0}$ the boundary of $\left.K\right)$. We approximate $K$ by a sequence of graphs $\Gamma_{m}$ with vertices $V_{m}$ and edge relation $x \underset{m}{\sim} y$ defined inductively by applying the $m$-fold iterations of $\left\{F_{i}\right\}$ to $V_{0}$. We define the renormalized graph energies

$$
\mathcal{E}_{m}(u)=\left(\frac{5}{3}\right)^{m} \sum_{x \widetilde{m}_{y}}(u(x)-u(y))^{2}
$$

for any function defined on $V_{m}$ (typically the restriction of a function defined on $K)$, and then

$$
\mathcal{E}(u)=\lim _{m \rightarrow \infty} \mathcal{E}_{m}(u)
$$

(the limit is always well defined since $\left\{\mathcal{E}_{m}(u)\right\}$ is always monotone increasing). We define the domain of energy $\operatorname{dom} E$ to be the functions $u$ with $\mathcal{E}(u)<\infty$. It is known that $\operatorname{dom} E \subseteq C(K)$, so these functions are determined by their restriction to $V_{*}=\bigcup_{m} V_{m}$, which is dense in $K$. The resistance metric $R(x, y)$ is defined by

$$
R(x, y)=(\inf \{\mathcal{E}(u): u(x)=0, u(y)=1\})^{-1}=\sup \frac{(u(x)-u(y))^{2}}{\mathcal{E}(u)}
$$

It is known that $R(x, y)$ is finite and defines a metric that is topologically equivalent to the Euclidean metric (it is metrically equivalent to a power of the Euclidean metric).

The pair $(\mathcal{E}, \operatorname{dom} E)$ satisfies the axioms for a Dirichlet form on $K$ : $\operatorname{dom} E$ modulo constants is a Hilbert space with respect to the inner product $\mathcal{E}(u, v)$ obtained from $\mathcal{E}(u)$ by polarization, and the Markov property holds. The energy is also local, meaning $\mathcal{E}(u, v)=0$ if $u$ and $v$ have disjoint support. It is known that the energy $\mathcal{E}(u)$ may be written as the integral over $K$ of a positive measure $\nu_{u}$, which may be characterized in two ways. First, if $C=F_{w} K$ is a cell $\left(F_{w}=F_{w_{1}} \circ F_{w_{2}} \circ \ldots \circ F_{w_{m}}\right.$ for any word $\left.w=\left(w_{1}, w_{2}, \ldots, w_{m}\right)\right)$, then $\nu_{u}(C)$ is defined in the same manner as $\mathcal{E}(u)$, except that the sum is restricted to edges lying in $C$. Second, for any $f \in \operatorname{dom} E$,

$$
\int f d \nu_{u}=\mathcal{E}(u, f u)-\frac{1}{2} \mathcal{E}\left(f, u^{2}\right) .
$$

Similarly, we can define signed measures $\nu_{u, v}$ so that $\mathcal{E}(u, v)=\int d \nu_{u, v}$. An interesting observation of Kusuoka ([11], see [2] for another proof) is that these 
measures are all singular with respect to the standard self-similar measure $\mu$ on $K$ (the normalized Hausdorff measure), but they are all absolutely continuous with respect to a single measure $\nu$, called the Kusuoka measure, defined below. See [5] and [6] for more recent singularity results.

A function on $K$ is called harmonic if it minimizes energy among all functions with the same boundary values. The space $\mathcal{H}_{0}$ of harmonic functions is 3-dimensional, as each harmonic function is determined by its boundary values. Indeed, there is a local linear harmonic extension algorithm

$$
\left.h\right|_{F_{w} V_{0}}=A_{w}\left(\left.h\right|_{V_{0}}\right)
$$

for $A_{w}=A_{w_{m}} \ldots A_{w_{1}}$, where $A_{i}$ are explicit matrices,

$$
A_{0}=\left(\begin{array}{ccc}
1 & 0 & 0 \\
\frac{2}{5} & \frac{2}{5} & \frac{1}{5} \\
\frac{2}{5} & \frac{1}{5} & \frac{2}{5}
\end{array}\right)
$$

and $A_{1}, A_{2}$ are obtained from $A_{0}$ by cyclic permutation of rows and columns. Of course constants are harmonic and have zero energy, so one can find an explicit orthonormal basis $\left\{h_{1}, h_{2}\right\}$ for harmonic functions in the energy inner product. Then we define

$$
\nu=\nu_{h_{1}}+\nu_{h_{2}} .
$$

It is easy to see that $\nu$ is independent of the choice of basis.

So now, given a conformal factor $\varphi$, we will define a conformal energy by

$$
\mathcal{E}_{\varphi}(u)=\int \varphi d \nu_{u}
$$

and

$$
\mathcal{E}_{\varphi}(u, v)=\int \varphi d \nu_{u, v}
$$

Using the ideas in [9], it is straightforward to verify that $\mathcal{E}_{\varphi}$ with domain $\operatorname{dom} E$ is a Dirichlet form that is strongly local and for which the resistance metric is finite. This is actually a nontrivial fact. In [13] a description of all such Dirichlet forms is given, but it is very indirect and does not allow for the construction of a large family of forms.

It follows from [9] that the energy $\mathcal{E}_{\varphi}$ may by obtained as a monotone increasing limit of graph energies $\left(\mathcal{E}_{\varphi}\right)_{m}$ on $\Gamma_{m}$,

$$
\left(\mathcal{E}_{\varphi}\right)_{m}(u)=\sum_{x \widetilde{m}_{y}} c_{m}(x, y)(u(x)-u(y))^{2}
$$

for certain positive conductances $c_{m}(x, y)$. In fact (1.12) is just the restriction of $\mathcal{E}_{\varphi}$ to $\Gamma_{m}$. That means that $(1.12)$ is just the minimum of $\mathcal{E}_{\varphi}(\widetilde{u})$ over all $\widetilde{u}$ that agree with $u$ on $V_{m}$. The exact determination of the conductances is a difficult problem. One of the main results of this paper is that we can write a kind of differential equation that determines these conductances. Nevertheless, it is easy 
to find a different family of discrete energies $\left(\widetilde{\mathcal{E}}_{\varphi}\right)_{m}$, given by $(1.12)$ with different conductances $\widetilde{c}_{m}(x, y)$ so that

$$
\mathcal{E}_{\varphi}(u)=\lim _{m \rightarrow \infty}\left(\widetilde{\mathcal{E}}_{\varphi}\right)_{m}(u)
$$

for $u \in \operatorname{dom} E$, but not necessarily monotonic, at least in the case that $\varphi$ is continuous or even piecewise continuous. One can take

$$
\widetilde{c}(x, y)=\frac{1}{2}(\varphi(x)+\varphi(y))\left(\frac{5}{3}\right)^{m},
$$

or better still we can replace the discrete average $\frac{1}{2}(\varphi(x)+\varphi(y))$ with an integral average of $\varphi$ over the $m$-cell with vertices $x$ and $y$, with respect to $\mu$ or $\nu$. This is certainly adequate for numerical approximations and is clearly consistent with (1.10)

To construct a Laplacian we need an energy form and a measure. For the standard Laplacian $\Delta$ on $S G$ we choose the energy (1.4) and the measure $\mu$. We define $u \in \operatorname{dom} E$ and $\Delta u=f$ to mean that $u \in \operatorname{dom} E, f \in C(K)$, and

$$
-\mathcal{E}(u, v)=\int f v d \mu
$$

for all $v \in \operatorname{dom}_{0} E$, where $\operatorname{dom}_{0} \mathcal{E}$ is defined to be the subspace of $\operatorname{dom} E$ of functions vanishing on the boundary. Similarly we define $\operatorname{dom}_{L^{2}} \Delta$ with the only difference that we only require $f \in L^{2}$. Then $-\Delta$ on $\operatorname{dom}_{L^{2}} \Delta$ becomes a positive selfadjoint operator under Dirichlet boundary conditions with compact inverse (or under Neumann boundary conditions with compact resolvant), and so has a discrete spectrum, with eigenfunctions belonging to $\operatorname{dom} D$. In fact the nature of the eigenvalues and eigenfunctions is known explicity via the method of spectral decimation of Fukushima and Shima ([4], [15], [16]). For functions in $\operatorname{dom} D$ there is also a pointwise formula for $\Delta u$ as a limit of a difference quotient

$$
\Delta u(x)=\lim _{m \rightarrow \infty} \frac{3}{2} 5^{m} \Delta_{m} u(x)
$$

for $x \in V_{*} \backslash V_{0}$, where

$$
\Delta_{m} u(x)=\sum_{y \underset{m}{x} x}(u(y)-u(x)) .
$$

To define a conformal Laplacian $\Delta_{\varphi}$ we want to modify (1.15) by replacing $\mathcal{E}$ by $\mathcal{E}_{\varphi}$ on the left side, and also changing the measure $\mu$ on the right side. In Riemannian geometry, when we make a conformal change of metric we also change the Riemannian measure by multiplying by a power of the conformal factor, so that leads us to expect that the new measure $\mu_{\phi}$ should be a power of $\varphi$ multiplying $\mu$. But what power? One way to answer this is to think about cells of different sizes in $K$. They are all topologically equivalent to $K$, but for an $m$-cell, the energy is boosted by a factor of $\left(\frac{5}{3}\right)^{m}$, while the measure is reduced by a factor of $\left(\frac{1}{3}\right)^{m}$. Since $\left(\frac{1}{3}\right)^{m}=\left(\left(\frac{5}{3}\right)^{m}\right)^{-\alpha}$ for

$$
\alpha=\frac{\log (3)}{\log (5 / 3)}
$$


this is consistent with

$$
d \mu_{\phi}=\varphi^{-\alpha} d \mu .
$$

With this choice, we define $u \in \operatorname{dom} \Delta_{\varphi}$ and $\Delta_{\varphi} u=f$ by

$$
-\mathcal{E}_{\varphi}(u, v)=\int f v d \mu_{\phi}
$$

In this paper we study the spectra of the conformal Laplacians $\Delta_{\varphi}$. Although the method of spectral decimation does not apply, we are able to prove spectral asymptotic results, which depend only on the distribution of values $\mu(\{x: \varphi(x) \leq$ $t\})$ of $\varphi$. We also show that certain peculiar features of the spectrum of the standard Laplacian, such as the existence of infinitely many large spectral gaps, persist in $\Delta_{\varphi}$ provided $\varphi$ does not vary too much $\left(c_{2} / c_{1}\right.$ is close to 1 in $\left.(1.1)\right)$. We also present graphical data from numerical approximations of lower portions of the spectra. Similar results for the spectrum of Schrödinger operators $-\Delta+V$ are given in [14].

In the second half of this paper we present a linear extension algorithm for computing energy measures of harmonic functions for the standard energy on $S G$. The definition of energy measure yields a quadratic expression for $\nu_{h}\left(F_{w} K\right)$ in terms of the matrix $A_{w}$ and the initial values $\left.h\right|_{V_{0}}$. Instead we work with the 3 -vectors $e(w)=\left(\nu_{h}\left(F_{w} F_{i} K\right)\right)_{i=0,1,2}$, where the initial vector corresponding to the empty word $e=\left(\nu_{h}\left(F_{0} K\right), \nu_{h}\left(F_{1} K\right), \nu_{h}\left(F_{2} K\right)\right)$ is a quadratic expression in $\left.h\right|_{V_{0}}$. We find another set of matrices $E_{i}$ such that

$$
e(w)=E_{w} e \text { for } \quad E_{w}=E_{w_{m}} \ldots E_{w_{1}} .
$$

The initial vector $e$ is not arbitrary, but must lie on a certain cone, which is preserved by the $E_{i}$ matrices. In other words, we have a complete description of the energy measure for a harmonic function in terms of a linear dynamical system on a cone in 3-space. We believe that this description will be very useful. As an application we give an algorithm for computing the $L^{p}$ dimension ([17]) of $\nu_{h}$ for integer values of $p \geq 2$ and find the explicit values for $p=2,3,4$. It follows from results in [2] that $\nu_{u}$ will have the same dimensions as $\nu_{h}$ for any $u \in \operatorname{dom} E$.

The organization of this paper is as follows. In section 2 we prove the differential equation for the conductances (and effective resistances) for conformal energies $\mathcal{E}_{\varphi}$. In section 3 we study the spectrum of the conformal Laplacian $\Delta_{\varphi}$. In section 4 we establish the linear extension algorithm for energy measures of harmonic functions. In section 5 we study the $L^{p}$ dimensions of energy measures. In section 6 we discuss extensions of the results in sections 4 and 5 to some other p.c.f. self-similar fractals.

A website with further details of our results is available at www.math.cornell. $\mathrm{edu} / \sim \mathrm{mhall} /$.

\section{Derivatives of CONFORMAl ENERGY}

In this section we study the differentiability of the conformal energy as a function of the conformal factor $\varphi(x)$ in the Banach space $C(K)$. As mentioned in the introduction, the energy $\mathcal{E}_{\varphi}$ determines conductances $c_{m}(x, y)$ for neighboring vertices $x, y$ in $V_{m}$, and we would like to understand how they depend on $\varphi$. In fact it suffices to understand what happens for $m=0$, since the problem is local on each cell $F_{w} K$. However the conductances $c_{0}\left(q_{i}, q_{j}\right)$ may be expressed in terms 
of effective resistances $R_{\varphi}\left(q_{i}, q_{j}\right)$ defined by $(1.5)$ with $\mathcal{E}$ replaced by $\mathcal{E}_{\varphi}$. Indeed, it is easy to see that

$$
R_{\varphi}\left(q_{1}, q_{2}\right)=\frac{c_{0}\left(q_{0}, q_{1}\right)+c_{0}\left(q_{2}, q_{0}\right)}{C}, \quad \text { etc. }
$$

for

$$
C=c_{0}\left(q_{0}, q_{1}\right) c_{0}\left(q_{1}, q_{2}\right)+c_{0}\left(q_{1}, q_{2}\right) c_{0}\left(q_{2}, q_{0}\right)+c_{0}\left(q_{2}, q_{0}\right) c_{0}\left(q_{0}, q_{1}\right) .
$$

We need to solve for the conductances. Taking linear combinations of (2.1) we obtain

$$
c_{0}\left(q_{1}, q_{2}\right)=\frac{C}{2}\left(R_{\varphi}\left(q_{0}, q_{1}\right)+R_{\varphi}\left(q_{2}, q_{0}\right)-R_{\varphi}\left(q_{1}, q_{2}\right)\right), \quad \text { etc. }
$$

Multiplying the equations (2.3) and adding we obtain an equation with $C$ on the left and $C^{2}$ on the right, which allows us to solve for $C$ and substitute the result back into (2.3) to obtain finally

$$
c_{0}\left(q_{1}, q_{2}\right)=\frac{2\left(\left(R_{\varphi}\left(q_{0}, q_{1}\right)+R_{\varphi}\left(q_{2}, q_{0}\right)-R_{\varphi}\left(q_{1}, q_{2}\right)\right)\right.}{D}, \quad \text { etc. }
$$

$$
\begin{aligned}
D=2\left(R_{\varphi}\left(q_{0}, q_{1}\right) R_{\varphi}\left(q_{1}, q_{2}\right)+\right. & R_{\varphi}\left(q_{1}, q_{2}\right) R_{\varphi}\left(q_{2}, q_{0}\right)+R_{\varphi}\left(q_{2}, q_{0}\right) R_{\varphi}\left(q_{0}, q_{1}\right) \\
& \left.-\left(R_{\varphi}\left(q_{0}, q_{1}\right)\right)^{2}-\left(R_{\varphi}\left(q_{1}, q_{2}\right)\right)^{2}-\left(R_{\varphi}\left(q_{2}, q_{0}\right)\right)^{2}\right) .
\end{aligned}
$$

So our goal will be to find the derivative of $R_{\varphi}$ with respect to $\varphi$. This question has a simple answer, and in fact we will be able to find $\frac{\partial}{\partial \varphi} R_{\varphi}(x, y)$ for any pair of points $x, y$. Moreover, the argument we give is quite generic and applies to any resistance form energy in the sense of [9]. Recall that $\frac{\partial}{\partial \varphi} R_{\varphi}(x, y)$ is defined to be an element of the dual space of $C(K)$, and we write

$$
\left\langle\frac{\partial}{\partial \varphi} R_{\varphi}(x, y), h\right\rangle=\lim _{t \rightarrow 0} t^{-1}\left(R_{\varphi+t h}(x, y)-R_{\varphi}(x, y)\right) .
$$

The differentiability of $R_{\varphi}$ means that the limit exists. We will also be able to identify the derivative as an energy measure.

Definition 2.1. Let $u_{\varphi}$ denote the energy minimizer in (1.5) for $\mathcal{E}_{\varphi}$, i.e. $u_{\varphi}(x)=$ $0, u_{\varphi}(y)=1$, and $u_{\varphi}$ minimizes $\mathcal{E}_{\varphi}(u)$ over all functions $u$ satisfying these two conditions. Similarly, let $u_{\varphi+t h}$ denote the energy minimizer for $\mathcal{E}_{\varphi+t h}$. This is well defined for $t$ small enough so that $\varphi+t h$ is positive.

Note that $u_{\varphi}$ is harmonic in the complement of $\{x, y\}$. In particular, if $x, y$ are boundary points, then $u_{\varphi}$ is a global harmonic function in $K$.

Theorem 2.2. Assume (1.1). Then $R_{\varphi}(x, y)$ is differentiable and

$$
\left\langle\frac{\partial}{\partial \varphi} R_{\varphi}(x, y), h\right\rangle=-R_{\varphi}(x, y)^{2} \int h d \nu_{\varphi}
$$

for any $h \in C(K)$. (Here $\nu_{u_{\varphi}}$ is defined with respect to the energy $\mathcal{E}$.)

Proof. Note that $R_{\varphi}(x, y)^{-1}=\mathcal{E}_{\varphi}\left(u_{\varphi}\right)$ and $\left(R_{\varphi+t h}\right)^{-1}=\mathcal{E}_{\varphi+t h}\left(u_{\varphi+t h}\right)$. Thus, by the quotient rule for derivatives, it suffices to show that

$$
\lim _{t \rightarrow 0} t^{-1}\left(\mathcal{E}_{\varphi+t h}\left(u_{\varphi+t h}\right)-\mathcal{E}_{\varphi}\left(u_{\varphi}\right)\right)=\int h d \nu_{\varphi}
$$


holds for all $h \in C(K)$. The idea of the proof is that we can replace $u_{\varphi+t h}$ by $u_{\varphi}$ in (2.8), making a change of order $o(t)$ that disappears in the limit, and we already have

$$
t^{-1}\left(\mathcal{E}_{\varphi+t h}\left(u_{\varphi}\right)-\mathcal{E}_{\varphi}\left(u_{\varphi}\right)\right)=\int h d \nu_{\varphi}
$$

even without taking the limit.

Observe that both $u_{\varphi}$ and $u_{\varphi+t h}$ satisfy $u(x)=0$ and $u(y)=1$, so by energy minimization we have

$$
\mathcal{E}_{\varphi+t h}\left(u_{\varphi+t h}\right)-\mathcal{E}_{\varphi+t h}\left(u_{\varphi}\right) \leq 0
$$

and

$$
\mathcal{E}_{\varphi}\left(u_{\varphi+t h}\right)-\mathcal{E}_{\varphi}\left(u_{\varphi}\right) \geq 0
$$

hence

$$
\begin{aligned}
\mathcal{E}_{\varphi+t h} & \left(u_{\varphi+t h}\right)-\mathcal{E}_{\varphi}\left(u_{\varphi+t h}\right) \\
& \leq \mathcal{E}_{\varphi+t h}\left(u_{\varphi+t h}\right)-\mathcal{E}_{\varphi}\left(u_{\varphi}\right) \\
& \leq \mathcal{E}_{\varphi+t h}\left(u_{\varphi}\right)-\mathcal{E}_{\varphi}\left(u_{\varphi}\right) .
\end{aligned}
$$

Since we can evaluate the outer expressions in (2.12) exactly, this means

$$
\begin{aligned}
t \int h d \nu_{u_{\varphi+t h}} & \leq \mathcal{E}_{\varphi+t h}\left(u_{\varphi+t h}\right)-\mathcal{E}_{\varphi}\left(u_{\varphi}\right) \\
& \leq t \int h d \nu_{u_{\varphi} .}
\end{aligned}
$$

Thus to complete the proof it suffices to show that

$$
\lim _{t \rightarrow 0} \int h d \nu_{u_{\varphi+t h}}=\int h d \nu_{u_{\varphi}} .
$$

We will establish (2.14) for $h \in \operatorname{dom} E$, and since $\operatorname{dom} E$ is dense in $C(K)$, it follows that it holds for all $h \in C(K)$; this uses the uniform boundedness of the measures $\nu_{\varphi+t h}$.

Now we claim that $u_{\varphi+t h}$ converges to $u_{\varphi}$ in energy as $t \rightarrow 0$; in fact,

$$
\mathcal{E}\left(u_{\varphi+t h}-u_{\varphi}\right) \leq c|t|
$$

for $h \in \operatorname{dom} E$. Since $\mathcal{E}_{\varphi}$ is equivalent to $\mathcal{E}$, it suffices to show the same estimate for $\mathcal{E}_{\varphi}$. Now $u_{\varphi+t h}-u_{\varphi}$ vanishes at $x$ and $y$, so it must be $\mathcal{E}_{\varphi}$-orthogonal to $u_{\varphi}$ by the minimization condition,

$$
\mathcal{E}_{\varphi}\left(u_{\varphi+t h}-u_{\varphi}, u_{\varphi}\right)=0
$$

(since $\mathcal{E}_{\varphi}\left(u_{\varphi}+s\left(u_{\varphi+t h}-u_{\varphi}\right)\right) \geq \mathcal{E}_{\varphi}\left(u_{\varphi}\right)$ for all $s$ ). Thus

$$
\begin{aligned}
\mathcal{E}_{\varphi}\left(u_{\varphi+t h}-u_{\varphi}\right)= & \mathcal{E}_{\varphi}\left(u_{\varphi+t h}\right)-\mathcal{E}_{\varphi}\left(u_{\varphi}\right) \\
=\left(\mathcal{E}_{\varphi}\left(u_{\varphi+t h}\right)\right. & \left.-\mathcal{E}_{\varphi+t h}\left(u_{\varphi+t h}\right)\right) \\
& +\left(\mathcal{E}_{\varphi+t h}\left(u_{\varphi+t h}\right)-\mathcal{E}_{\varphi+t h}\left(u_{\varphi}\right)\right) \\
& +\left(\mathcal{E}_{\varphi+t h}\left(u_{\varphi}\right)-\mathcal{E}_{\varphi}\left(u_{\varphi}\right)\right) .
\end{aligned}
$$

Then (2.10) allows us to drop the middle term to obtain

$$
\mathcal{E}_{\varphi}\left(u_{\varphi+t h}-u_{\varphi}\right) \leq\left(\mathcal{E}_{\varphi}\left(u_{\varphi+t h}\right)-\mathcal{E}_{\varphi+t h}\left(u_{\varphi+t h}\right)\right)+\left(\mathcal{E}_{\varphi+t h}\left(u_{\varphi}\right)-\mathcal{E}_{\varphi}\left(u_{\varphi}\right)\right)
$$


However, by (1.6) and (1.10) we have

$$
\mathcal{E}_{\varphi+t h}(u)-\mathcal{E}_{\varphi}(u)=t\left(\mathcal{E}(u, h u)-\frac{1}{2} \mathcal{E}\left(h, u^{2}\right)\right)
$$

for any $u$, and it is easy to see that

$$
\left|\mathcal{E}(u, h u)-\frac{1}{2} \mathcal{E}\left(h, u^{2}\right)\right| \leq c \mathcal{E}(u)
$$

if $u(x)=0$. Since $\mathcal{E}\left(u_{\varphi+t h}\right)$ is uniformly bounded for small $t$, we may combine $(2.20),(2.19)$, and (2.18) to obtain (2.15) (for $\mathcal{E}_{\varphi}$ ) as claimed.

Returning to (2.14), we have by (1.6) that

$$
\begin{gathered}
\int h d \nu_{u_{\varphi+t h}}-\int h d \nu_{u}=\left(\mathcal{E}\left(u_{\varphi+t h}, h u_{\varphi+t h}\right)-\mathcal{E}\left(u_{\varphi+t h}, h u_{\varphi}\right)\right) \\
-\frac{1}{2}\left(\mathcal{E}\left(h, u_{\varphi+t h}^{2}\right)-\mathcal{E}\left(h, u_{\varphi}^{2}\right)\right) .
\end{gathered}
$$

It is easy to control each of the differences in $(2.21)$ by $\mathcal{E}\left(u_{\varphi+t h}-u_{\varphi}\right)$, and hence (2.15) implies

$$
\left|\int h d \nu_{u_{\varphi+t h}}-\int h d \nu_{u_{\varphi}}\right| \leq c|t| .
$$

It is easy to see that the result extends to functions $h$ that are piecewise continuous on $m$-cells, since the argument can be localized to each cell. It seems plausible that it is valid for all $h \in L^{\infty}(d \nu)$, but it is not clear how to prove this.

\section{SpeCtral asymptotics of CONFORMal Laplacians}

In this section we show how to transfer information about the spectrum of the standard Laplacian on $S G$ to information about the spectrum of the conformal Laplacian $\Delta_{\varphi}$. We may impose either Dirichlet or Neumann boundary conditions. Let $\left\{\lambda_{j}\right\}$ denote the eigenvalues in increasing order (repeated according to multiplicity) of $\Delta$, and $\left\{\widetilde{\lambda}_{j}\right\}$ the same for $\Delta_{\varphi}$. Let $N(x)=\#\left\{\lambda_{j} \leq x\right\}$ and $\widetilde{N}(x)=\#\left\{\widetilde{\lambda}_{j} \leq x\right\}$ denote the eigenvalue counting functions. It is known that $N(x)$ has a growth rate of $x^{\beta}$ for $\beta=\log (3) / \log (5)$. This power may be interpreted as the ratio $\alpha /(\alpha+1)$, where $\alpha=\log 3 / \log (5 / 3)$ is the Hausdorff dimension of $S G$ in the resistance metric, and $\alpha+1=\log 5 / \log (5 / 3)$ is the order of the operator $\Delta([19])$. What is more interesting is that there is a more refined statement $([10])$ :

$$
\frac{N(x)}{x^{\beta}}=\psi(\log x)+R(x)
$$

with $R(x) \rightarrow 0$ as $x \rightarrow \infty$, where $\psi$ is a certain discontinuous periodic function of period $\log 5$, satisfying

$$
0<c_{3} \leq \psi \leq c_{4}
$$

In fact $\psi$ has a countable number of jump discontinuities, with only a finite number with jump size exceeding $\epsilon$ for any fixed $\epsilon>0$. Its graph is shown in Figure 1. (More precisely, this is the graph of the spectral counting function for a finite graph approximation to $S G$, and this approximation to the $S G$ spectral 


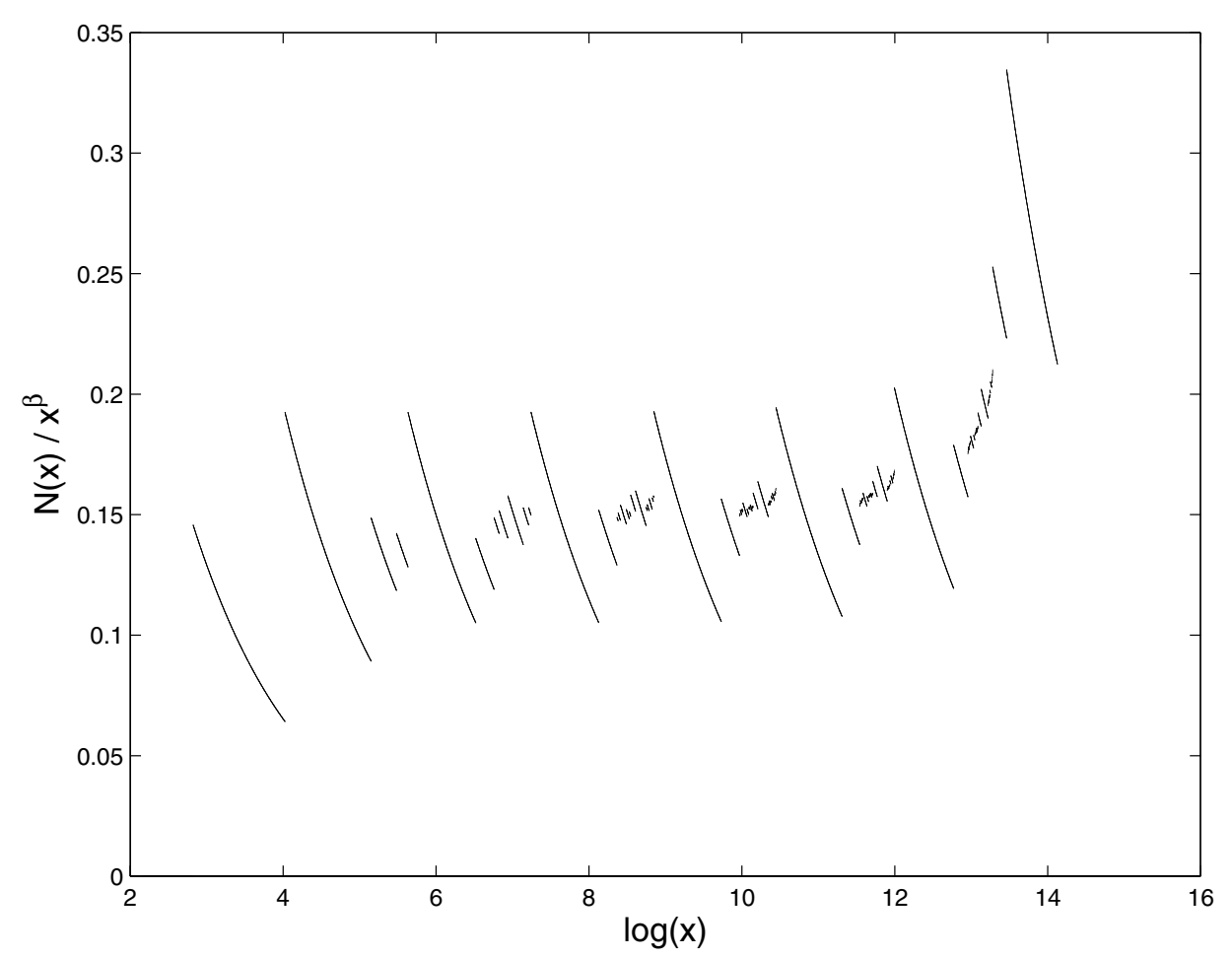

Figure 1. The Weyl ratio for the spectral counting function of the standard Laplacian as a function of $\log x$

counting function loses accuracy for large $x$.) It is also known that $R(x)=O\left(x^{-\beta}\right)$, but we will not use this fact, and the analogous statement for $\widetilde{N}(x)$ is most likely not true. We will use the fact that (3.1) continues to hold if we only count localized eigenfunctions. Fix a value $m$, and let $N_{m}(x)$ denote the number of eigenvalues $\lambda_{j} \leq x$ corresponding to eigenfunctions supported in any one of the $m$-cells $F_{w} K$ with $|w|=m$. Then (3.1) holds for $N_{m}(x)$ in place of $N(x)$, with the same function $\psi$ (but a different remainder). In other words the nonlocalized eigenfunctions are relatively rare,

$$
N(x)-N_{m}(x)=o\left(x^{\beta}\right)
$$

(in fact it grows with a smaller power).

Now suppose $\varphi$ is piecewise constant on $m$-cells, say

$$
\varphi=\sum_{|w|=m} a_{w} \chi_{F_{w} K}
$$

If $u$ is an eigenfunction of $\Delta$ supported on $F_{w} K$ with eigenvalue $\lambda$, then it is also an eigenfunction of $\Delta_{\varphi}$ with eigenvalue $a_{w}^{(1+\alpha)} \lambda$. If we write $\widetilde{N}_{m}(x)$ for the counting function for localized eigenfunctions of $\Delta_{\varphi}$, it is easy to see that the analog of (3.3) holds, so

$$
\frac{\widetilde{N}(x)}{x^{\beta}}=\frac{\widetilde{N}_{m}(x)}{x^{\beta}}+R(x) .
$$


We may compute $\widetilde{N}_{m}(x)$ exactly because it is known that the number of eigenfunctions supported in $F_{w} K$ is proportional to $\mu\left(F_{w} K\right)=3^{-m}$ (this is exactly true in the Dirichlet case, and off by an inconsequential amount for the three boundary cells in the Neumann case). Thus

$$
\tilde{N}_{m}(x)=\sum_{|w|=m} \mu\left(F_{w} K\right) \cdot N_{m}\left(a_{w}^{1+\alpha} x\right) .
$$

When we substitute (3.1) into (3.6) we obtain $($ since $(1+\alpha) \beta=\alpha)$

$$
\frac{\tilde{N}(x)}{x^{\beta}}=\sum_{|w|=m} \mu\left(F_{w} K\right) \cdot a_{w}^{\alpha}\left(\psi\left(\log x+(\alpha+1) \log a_{w}\right)\right)+R(x) .
$$

Note that we can write the sum in (3.7) as

$$
\int \varphi(y)^{\alpha} \psi(\log x+(\alpha+1) \log \varphi(y)) d \mu(y)
$$

and this expression makes sense for any conformal factor $\varphi$. We will define the function $\psi_{\varphi}(\log x)$ by (3.8). Note that (3.8) is essentially a convolution; by writing $\varphi(y)=t$ and defining the pull-back measure $\mu \circ \varphi^{-1}$ on the line, we have

$$
\psi_{\varphi}(\log x)=\int_{c_{1}}^{c_{2}} t^{\alpha} \psi(\log x+(\alpha+1) \log t) d \mu \circ \varphi^{-1}(t)
$$

The basic hypothesis we will make is that the measure $\mu \circ \varphi^{-1}$ is absolutely continuous. Then, after a change of variable, (3.9) is a convolution of an $L^{1}$ function and an $L^{\infty}$ function, hence continuous. Of course, this hypothesis is not satisfied by the piecewise constant function (3.4).

Theorem 3.1. Assume $\varphi$ is piecewise continuous, satisfies (1.1), and $\mu \circ \varphi^{-1}$ is absolutely continuous. Then

$$
\frac{\widetilde{N}(x)}{x^{\beta}}=\psi_{\varphi}(\log x)+\widetilde{R}(x)
$$

with $\lim _{x \rightarrow \infty} \widetilde{R}(x)=0$.

Proof. Given any $\epsilon>0$, there exists $\delta>0$ so that $\left|t-t^{\prime}\right| \leq \delta$ implies $\mid \psi_{\varphi}(t)-$ $\psi_{\varphi}\left(t^{\prime}\right) \mid \leq \epsilon$, since $\psi_{\varphi}$ is continuous. Next choose $\varphi_{1}$ of the form (3.4) (for some $m$ ) so that $\left\|\varphi-\varphi_{1}\right\|_{\infty} \leq \delta$ and also $\left\|\psi_{\varphi}-\psi_{\varphi_{1}}\right\| \leq \epsilon$ (this uses the piecewise continuity of $\varphi$ for the first estimate, and simple estimates using (3.9) for the second).

Now we want to compare $\widetilde{N}(x)$ and $N^{\prime}(x)$, where $N^{\prime}(x)$ denotes the counting function for $\Delta_{\varphi_{1}}$. Because of (1.1) we may write

$$
\left(1-\delta^{\prime}\right) \varphi_{1}(x) \leq \varphi(x) \leq\left(1+\delta^{\prime}\right) \varphi_{1}(x)
$$

where $\delta^{\prime}$ may be controlled by $\delta$. Then $N^{\prime}\left(\left(1-\delta^{\prime}\right)^{1+\alpha} x\right) \leq \widetilde{N}(x) \leq N^{\prime}\left(\left(1+\delta^{\prime}\right)^{1+\alpha} x\right)$, which may be written

$$
N^{\prime}\left(\left(1-\delta^{\prime \prime}\right) x\right) \leq \widetilde{N}(x) \leq N^{\prime}\left(\left(1+\delta^{\prime \prime}\right) x\right)
$$


with $\delta^{\prime \prime}$ controlled by $\delta$. So

$$
\begin{aligned}
\frac{\tilde{N}(x)}{x^{\beta}} \leq & \left(1+\delta^{\prime \prime}\right)^{\beta} \frac{\left.N^{\prime}\left(1+\delta^{\prime \prime}\right) x\right)}{\left(\left(1+\delta^{\prime \prime}\right) x\right)^{\beta}} \\
= & \left(1+\delta^{\prime \prime}\right)^{\beta}\left(\psi_{\varphi_{1}}\left(\log x+\log \left(1+\delta^{\prime \prime}\right)\right)+R^{\prime}(x)\right) \\
= & \left(1+\delta^{\prime \prime}\right)^{\beta}\left(\psi_{\varphi}(\log x)+\left[\psi_{\varphi}\left(\log x+\log \left(1+\delta^{\prime \prime}\right)\right)-\psi_{\varphi}(\log x)\right]\right. \\
& \left.\quad+\left[\psi_{\varphi_{1}}\left(\log x+\log \left(1+\delta^{\prime \prime}\right)\right)-\psi_{\varphi}\left(\log \left(1+\delta^{\prime \prime}\right)\right)\right]+R^{\prime}(x)\right) .
\end{aligned}
$$

Now the two terms in brackets are each bounded by $\epsilon$, so we obtain

$$
\frac{\tilde{N}(x)}{x^{\beta}} \leq\left(1+\delta^{\prime \prime}\right)^{\beta}\left[\psi_{\varphi}(\log x)+2 \epsilon+R^{\prime}(x)\right]
$$

and a similar estimate from below. By taking $x$ large enough we can make $R^{\prime}(x) \leq \epsilon$ as well, so

$$
\left|\frac{\tilde{N}(x)}{x^{\beta}}-\left(1+\delta^{\prime \prime}\right)^{\beta} \psi_{\varphi}(\log x)\right| \leq\left(1+\delta^{\prime \prime}\right)^{\beta} 3 \epsilon,
$$

and if $\delta$ is small enough this yields $\left|\frac{\widetilde{N}(x)}{x^{\beta}}-\psi_{\varphi}(\log x)\right| \leq 5 \epsilon$ if $x$ is large enough.

It is striking that this is a better result than (3.1) because $\psi_{\varphi}$ is continuous. Also note that $\psi_{\varphi}$ only depends on $\mu \circ \varphi^{-1}$, which is determined by the distribution of values of $\varphi$ (say $\mu(\{x: \varphi(x) \geq s\}))$ and not the locations where the values are attained. So any measure-preserving permutation of $\varphi$ does not influence the spectral asymptotics. Note that we no longer expect to see any localized eigenfunctions of $\Delta_{\varphi}$, although there may be many eigenfunctions that are close to being localized.

Another striking and important feature of the spectrum of $\Delta$ is the existence of infinitely many large gaps, meaning places where

$$
\lambda_{j+1} \geq a \lambda_{j}
$$

for fixed $a>1$. (In fact it is possible to take $a \approx 2.425$.) We claim that this property persists for $\Delta_{\varphi}$ provided the ratio $c_{2} / c_{1}$ in (1.1) is close enough to 1 . To see this we need only recall the minimax formula:

$$
\lambda_{j}=\min _{\operatorname{dim} L=j} \max _{u \in L} R(u)
$$

for the Rayleigh quotient

$$
R(u)=\frac{\mathcal{E}(u)}{\int|u|^{2} d \mu}
$$

and of course the same formula holds for $\widetilde{\lambda}_{j}$ if we replace $R(u)$ by

$$
\widetilde{R}(u)=\frac{\mathcal{E}_{\varphi}(u)}{\int|u|^{2} d \mu_{\varphi}} .
$$

(This is the formula for Neumann spectra; for Dirichlet spectra we just need to impose Dirichlet conditions on the spaces $L$.) From (1.1) we easily obtain

$$
c_{1} \mathcal{E}(u) \leq \mathcal{E}_{\varphi}(u) \leq c_{2} \mathcal{E}(u),
$$

and also (1.19) implies

$$
c_{2}^{-\alpha} \int|u|^{2} d \mu \leq \int|u|^{2} d \mu_{\varphi} \leq c_{1}^{-\alpha} \int|u|^{2} d \mu .
$$


Together (3.18) and (3.19) imply

$$
c_{1}^{1+\alpha} R(u) \leq \widetilde{R}(u) \leq c_{2}^{1+\alpha} R(u),
$$

so the minimax formula implies

$$
c_{1}^{1+\alpha} \lambda_{j} \leq \widetilde{\lambda}_{j} \leq c_{2}^{1+\alpha} \lambda_{j}
$$

Combining (3.21) with (3.14) yields

$$
\widetilde{\lambda}_{j+1} \geq \widetilde{a} \lambda_{j}
$$

for

$$
\widetilde{a}=\left(\frac{c_{1}}{c_{2}}\right)^{1+\alpha} a .
$$

So the condition

$$
\frac{c_{2}}{c_{1}}<a^{1 /(1+\alpha)} \approx 1.325
$$

suffices to conclude that the spectrum of $\Delta_{\varphi}$ has infinitely many large gaps at exactly the same locations as the spectrum of $\Delta$. We can even ensure that $\widetilde{a}>2$ by further restricting

$$
\frac{c_{2}}{c_{1}}<\left(\frac{a}{2}\right)^{1 /(1+\alpha)} \approx 1.063 .
$$

In [21] it was shown that eigenfunction expansions (for $\Delta$ ) of continuous functions always converge uniformly to the function, provided you restrict to partial sums up to a large gap. The argument required both the existence of large spectral gaps and the known sub-Gaussian heat kernel estimates for $\Delta$. The same heat kernel estimates also hold for $\Delta_{\varphi}$ by recent results of Barlow, Bass, and Kumagai ([1]), so we have the same convergence theorem for eigenfunction expansions for $\Delta_{\varphi}$ provided (3.24) holds. Also by reasoning in [21], we can extend the result to products $K \times K$ of $S G$ ([20]) under the stronger condition (3.25). It is even possible to allow different functions $\varphi$ on each factor, provided (3.25) holds for each one. (In this context it would be more natural to allow conformal factors not necessarily of product form, but we will not discuss this here.)

Another interesting feature of the spectrum of $\Delta$ is that the set of all ratios $\left\{\lambda_{j} / \lambda_{k}\right\}$ of eigenvalues has gaps ([3],[22]). Again (3.12) guarantees that this will persist for the ratios $\left\{\widetilde{\lambda}_{j} / \widetilde{\lambda}_{k}\right\}$ of eigenvalues of $\Delta_{\varphi}$ provided $c_{2} / c_{1}$ is sufficiently small. Specifically, $2.0611106 \geq \lambda_{j} / \lambda_{k}$ or $2.428766 \leq \lambda_{j} / \lambda_{k}$, so $2.0611106\left(\frac{c_{2}}{c_{1}}\right)^{1+\alpha} \geq$ $\widetilde{\lambda}_{j} / \widetilde{\lambda}_{k}$ or $2.428766\left(\frac{c_{1}}{c_{2}}\right)^{1+\alpha} \leq \widetilde{\lambda}_{j} / \widetilde{\lambda}_{k}$, and this is a gap provided

$$
\left(\frac{c_{2}}{c_{1}}\right) \leq\left(\frac{2.428766}{2.0611106}\right)^{\frac{1}{2(1+\alpha)}} \approx 1.026 .
$$

To illustrate the above results, we produce, for various choices of the conformal factor $\varphi$, approximations of the spectrum of $\Delta_{\varphi}$ of the type in Figure 1. To form $\varphi$ we choose a perturbative function $h$ (which we choose to be an asymmetric sinusoidal function on a planar embedding of $S G$ ) and look at $\Delta_{\varphi}$ for $\varphi=1+$ th for different values of $t$. Thus we are able to adjust the ratio $\frac{c_{2}}{c_{1}}$ by varying $t$.

The graph in Figure 1 is an approximation of the logarithmic plot of the Weyl ratio $N(x) / x^{\beta}$, where $N$ is the spectral counting function, using a finite graph 
approximation of $S G$ with $m=7$. The graph of the Weyl ratio is asymptotic to a multiplicatively periodic function $\psi_{\varphi}$, so we attempt to surmise the nature of $\psi$ from our approximate plot, observing its nearly-periodic qualities. Figures $2-6$ use $\left(\Delta_{\varphi}\right)_{m}$ for $m=7$ also. The form of the calculations is similar to the pointwise formula (1.16). Here we instead use

$$
\Delta_{\varphi} u(x)=\lim _{m \rightarrow \infty} \frac{3}{2} 5^{m}\left(\Delta_{\varphi}\right)_{m} u(x)
$$

where

$$
\left(\Delta_{\varphi}\right)_{m} u(x)=\sum_{y \underset{m}{x}} \gamma_{x y}^{-(1+\alpha)}(u(y)-u(x))
$$

and the coefficients $\gamma_{x y}$ are obtained by using a step function approximation of $\varphi$. In this context it is easy to see that, for functions $v$ (in the sense of (1.20)) whose support is contained in some region where a given $\varphi$ takes some constant value $\gamma$, and for $u \in \operatorname{dom} \Delta_{\varphi}$, we have, by pulling out powers of gamma from $\mathcal{E}_{\varphi}$ and $d \mu_{\varphi}$ in $(1.20)$,

$$
-\mathcal{E}(u, v)=\gamma^{-(1+\alpha)} \int\left(\Delta_{\varphi} u\right) v d \mu
$$

The pointwise formulas (1.16) and (1.17) then imply that $\left(\Delta_{\varphi}\right)_{m}$ as defined above must converge to $\Delta_{\varphi}$.

In Figure 1 we are able to notice eigenvalues of high multiplicity and gaps in the spectrum of $\Delta$, corresponding to jump discontinuities and smooth, decreasing, concave up intervals of the Weyl ratio for $N(x)$, respectively. We observe in Figure 2 that for an only slightly nonconstant conformal factor (here we choose $\frac{c_{2}}{c_{1}}=$ 1.005) the graph of the Weyl ratio for $\tilde{N}$ is similar to that for $N$. The intervals of smoothness (indicating spectral gaps) persist, while instead of eigenvalues of high multiplicity we see large clusters of similar eigenvalues, producing rapid but fragmented increases instead of the large jumps observed in Figure 1. The second image is a closeup of several of the later periods.

As we increase the size of the perturbation, most gaps in the spectrum are destroyed, though the largest of these can be seen to persist for larger perturbations. Figures 3-6 show the cases for $\frac{c_{2}}{c_{1}}=1.02,1.1,1.15$, and 1.25 . It is more difficult to see the exact nature of the periodic function $\psi_{\varphi}$ as the perturbations increase since our approximation is only useful for the lower part of the spectrum of $\Delta_{\varphi}$. For a more slowly vanishing error function $\tilde{R}(x)$ we do not see several consecutive intervals of behavior closely resembling one another (cf. Figure 6).

As noted above, the function $\psi_{\varphi}$ depends only on the distribution of values of $\varphi$. Figures $7-11$ repeat the methods used for Figures $2-6$ with a new conformal factor obtained by composing with a random permutation of the level 3 cells of $S G$ (i.e. we use $\varphi^{\prime}=\varphi \circ \sigma$ and $\sigma: S G \rightarrow S G$ permutes the cells $F_{w}(S G)$, where $w$ runs over words of length 3 ).

More Weyl ratio plots of the type seen in Figures 1-11 can be found at www.math. cornell.edu $/ \sim$ mhall $/$. 

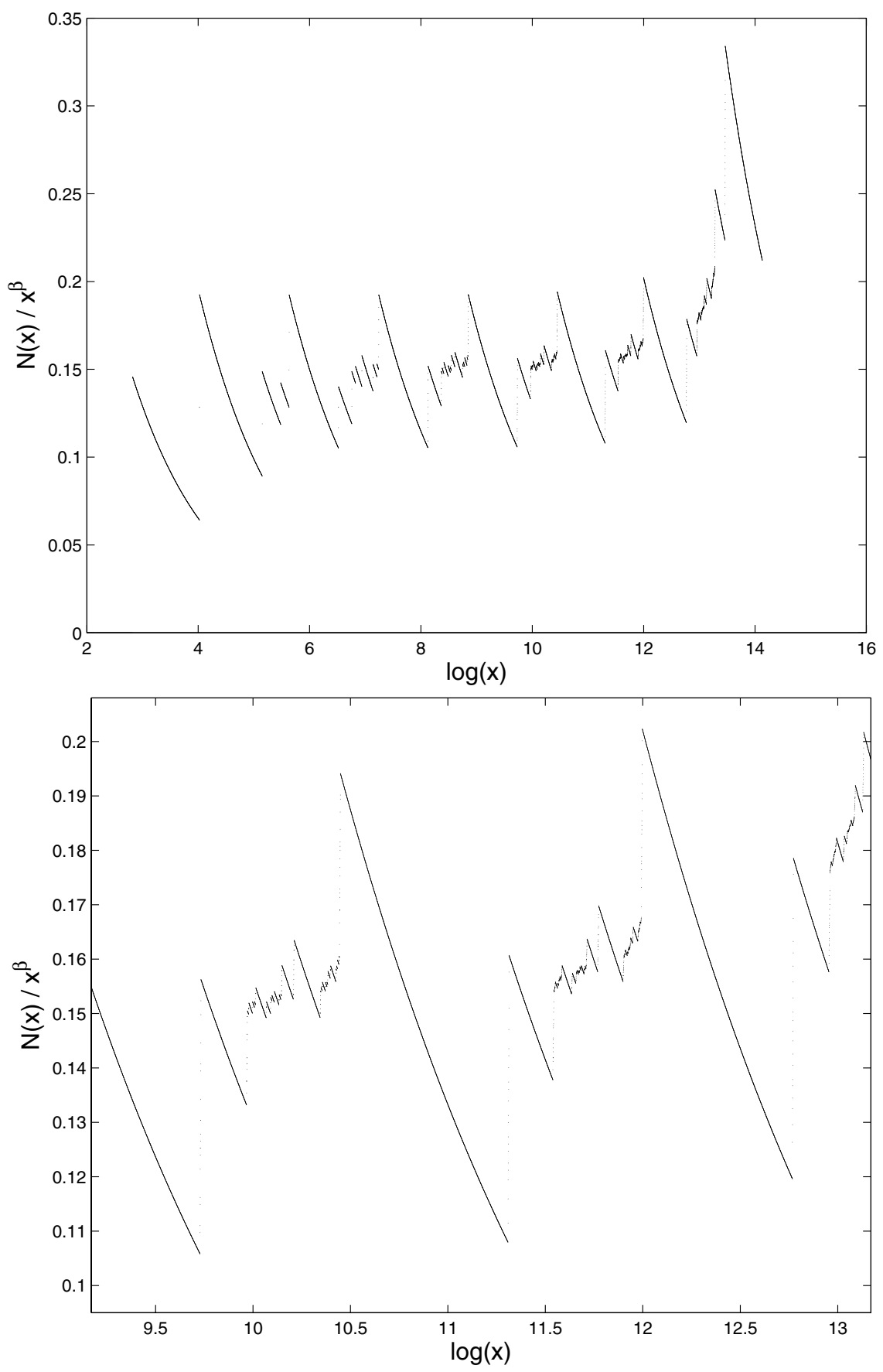

Figure 2. The Weyl ratio for the spectral counting function of $\Delta_{\varphi}$ with factor $c_{1} \leq \varphi \leq c_{2}$ such that $\frac{c_{2}}{c_{1}}=1.005$, as a function of $\log x$ 

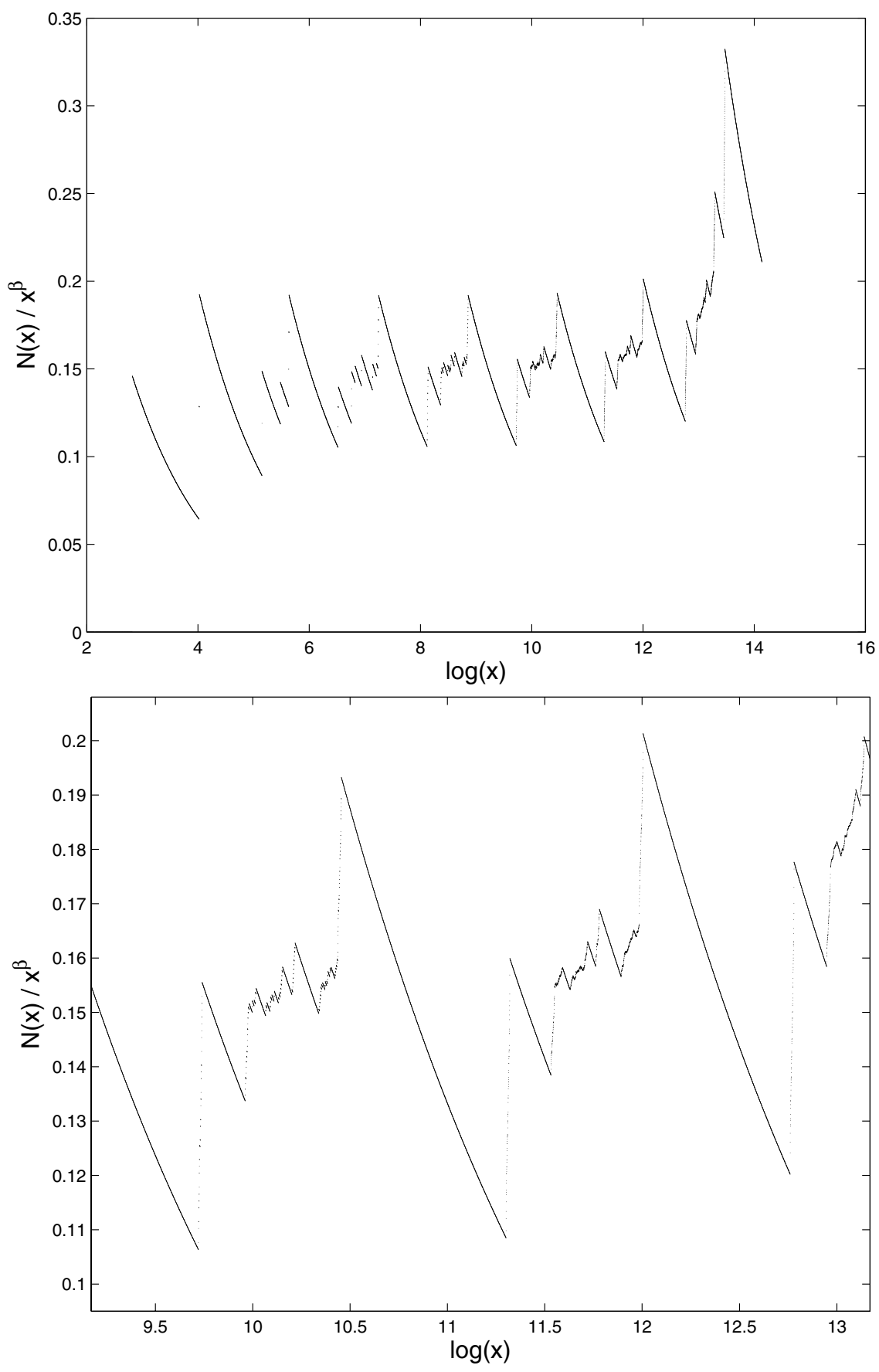

FIgURE 3. The Weyl ratio for the spectral counting function of $\Delta_{\varphi}$ with factor $c_{1} \leq \varphi \leq c_{2}$ such that $\frac{c_{2}}{c_{1}}=1.02$, as a function of $\log x$ 

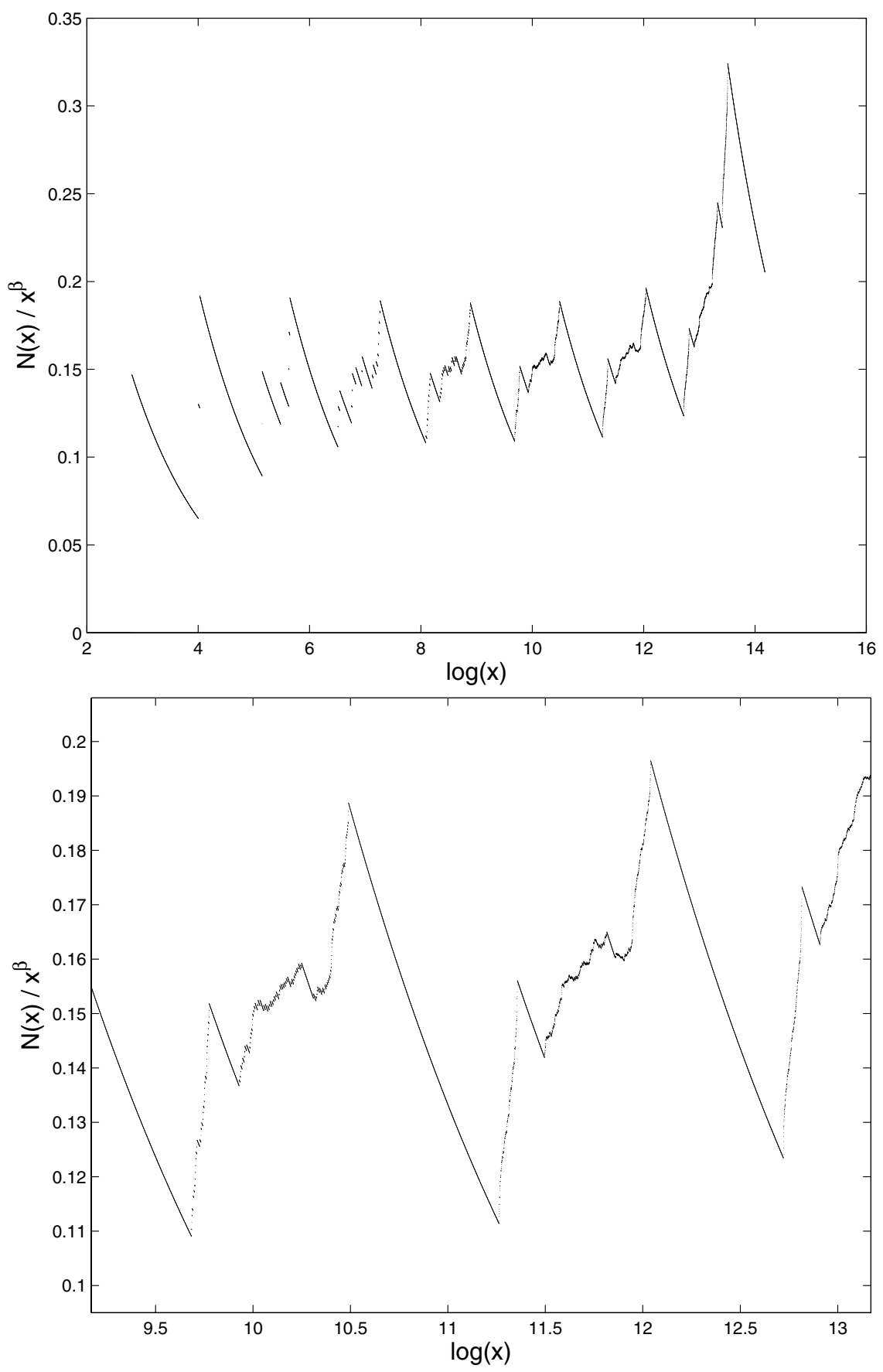

FIgURE 4. The Weyl ratio for the spectral counting function of $\Delta_{\varphi}$ with factor $c_{1} \leq \varphi \leq c_{2}$ such that $\frac{c_{2}}{c_{1}}=1.1$, as a function of $\log x$ 

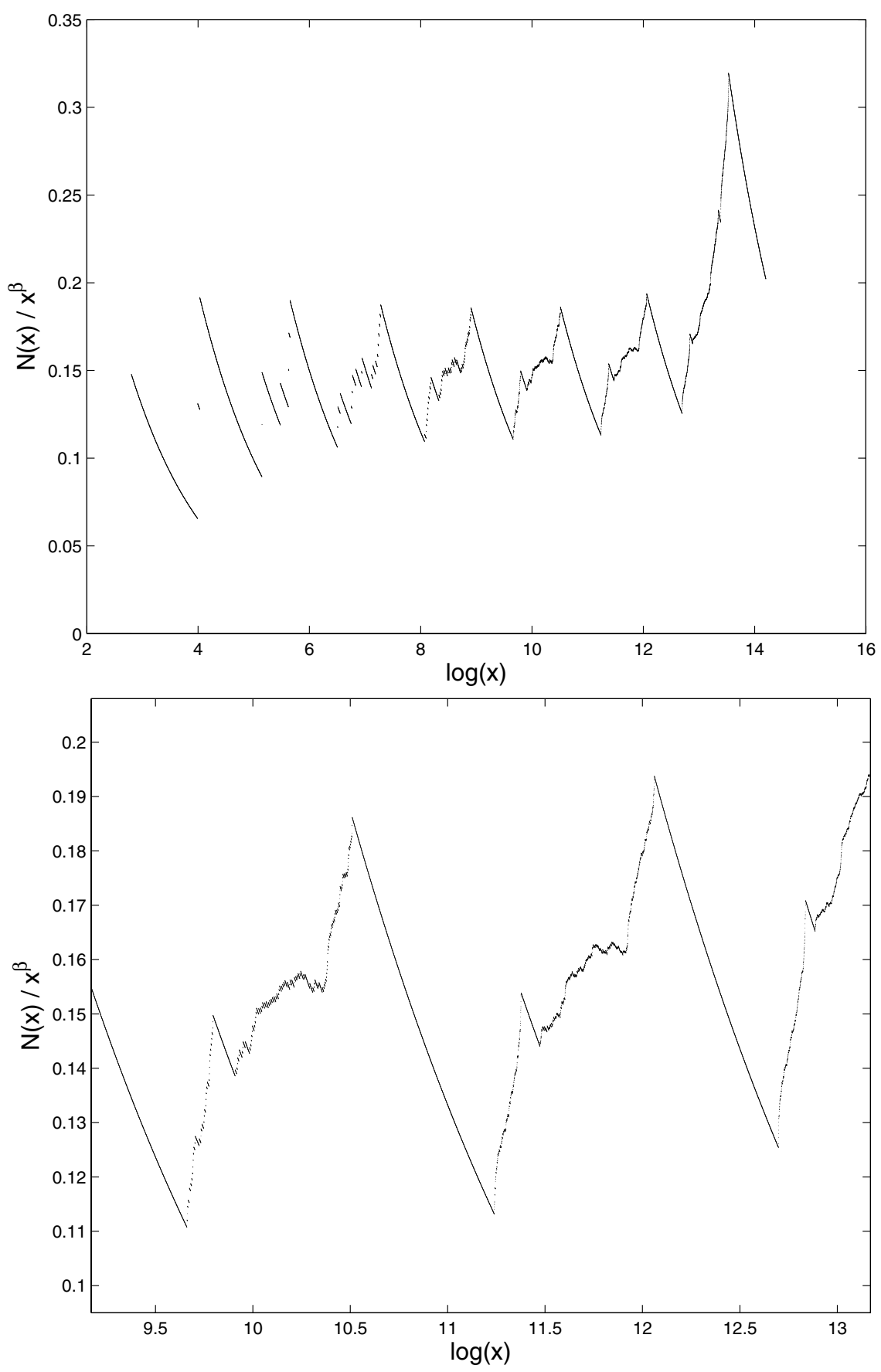

Figure 5. The Weyl ratio for the spectral counting function of $\Delta_{\varphi}$ with factor $c_{1} \leq \varphi \leq c_{2}$ such that $\frac{c_{2}}{c_{1}}=1.15$, as a function of $\log x$ 

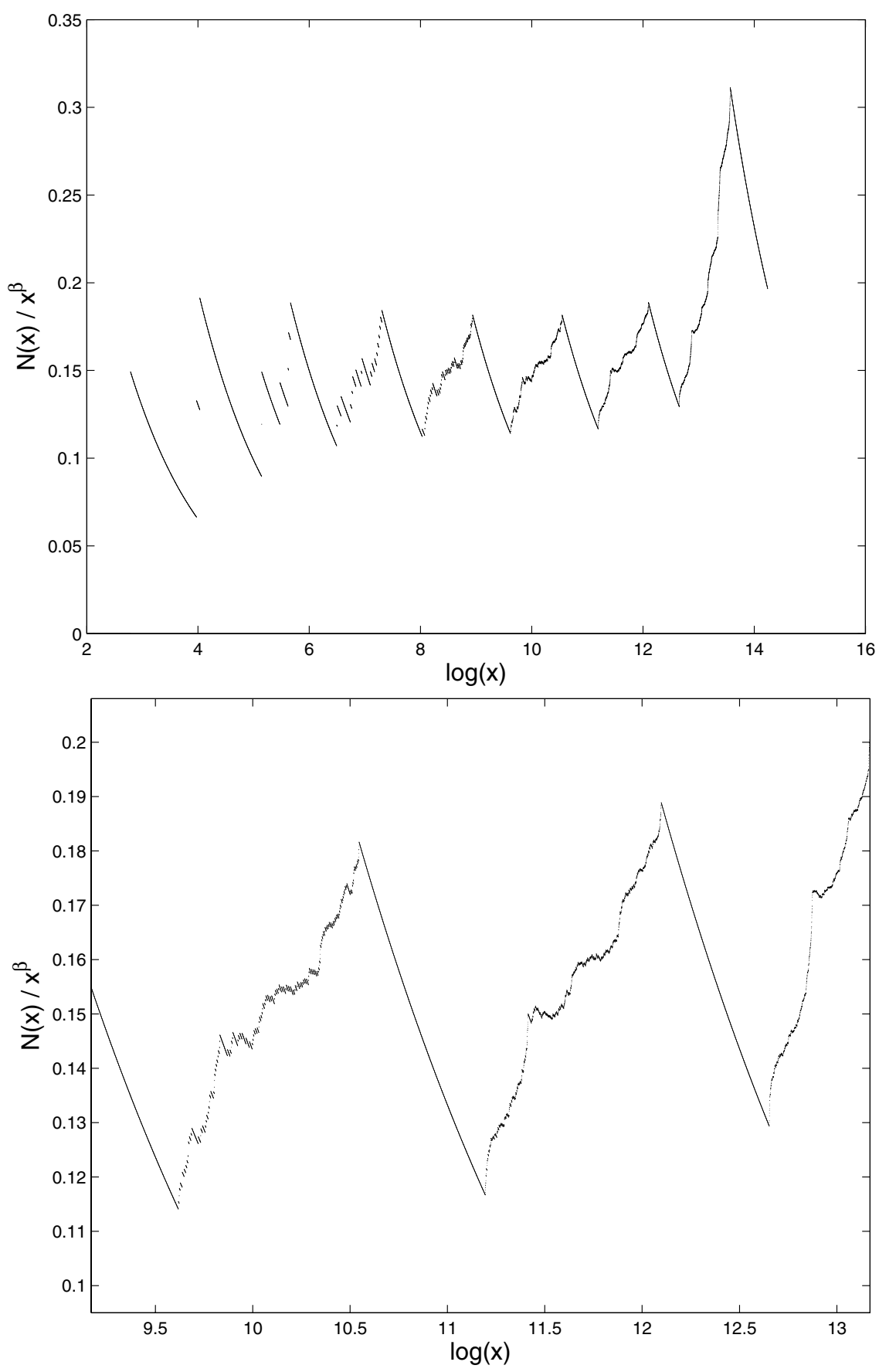

Figure 6. The Weyl ratio for the spectral counting function of $\Delta_{\varphi}$ with factor $c_{1} \leq \varphi \leq c_{2}$ such that $\frac{c_{2}}{c_{1}}=1.25$, as a function of $\log x$ 

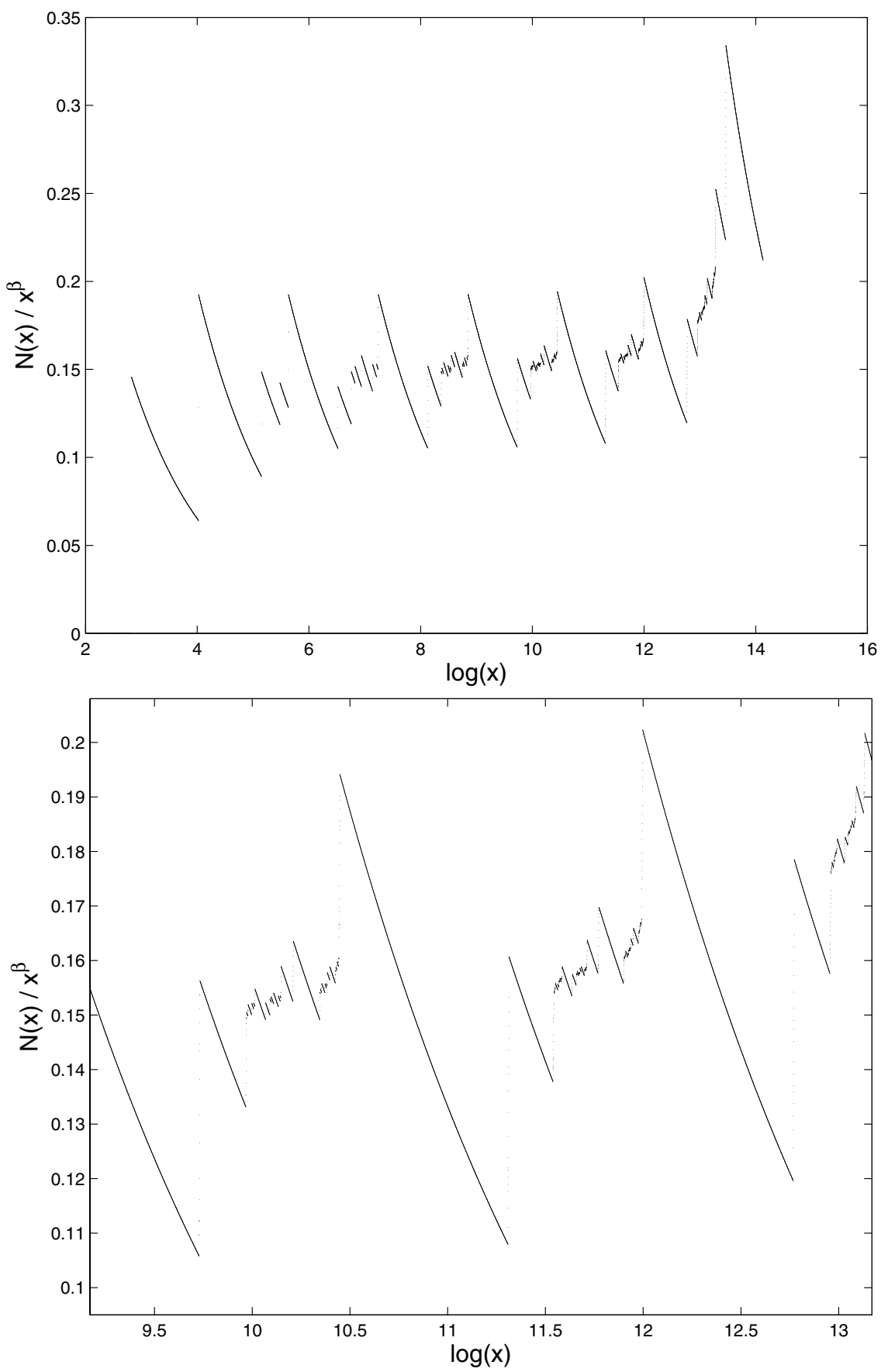

FIgURE 7. The Weyl ratio for the spectral counting function of $\Delta_{\varphi^{\prime}}$ with $\varphi^{\prime}=\varphi \circ \sigma$ such that $c_{1} \leq \varphi \leq c_{2}, \frac{c_{2}}{c_{1}}=1.005$, and $\sigma$ permutes the level 3 cells of $S G$, as a function of $\log x$ 

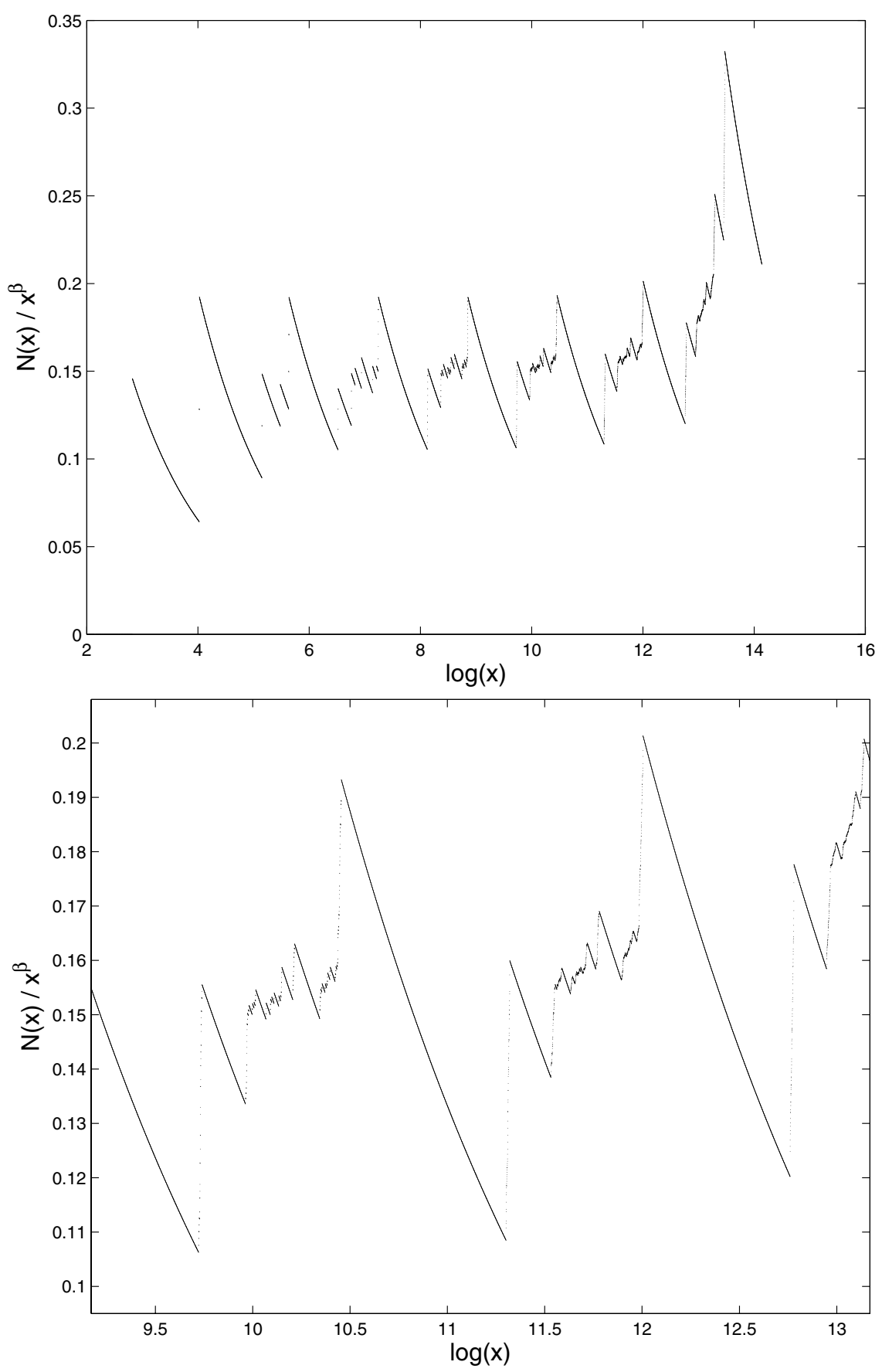

Figure 8. The Weyl ratio for the spectral counting function of $\Delta_{\varphi^{\prime}}$ with $\varphi^{\prime}=\varphi \circ \sigma$ such that $c_{1} \leq \varphi \leq c_{2}, \frac{c_{2}}{c_{1}}=1.02$, and $\sigma$ permutes the level 3 cells of $S G$, as a function of $\log x$ 

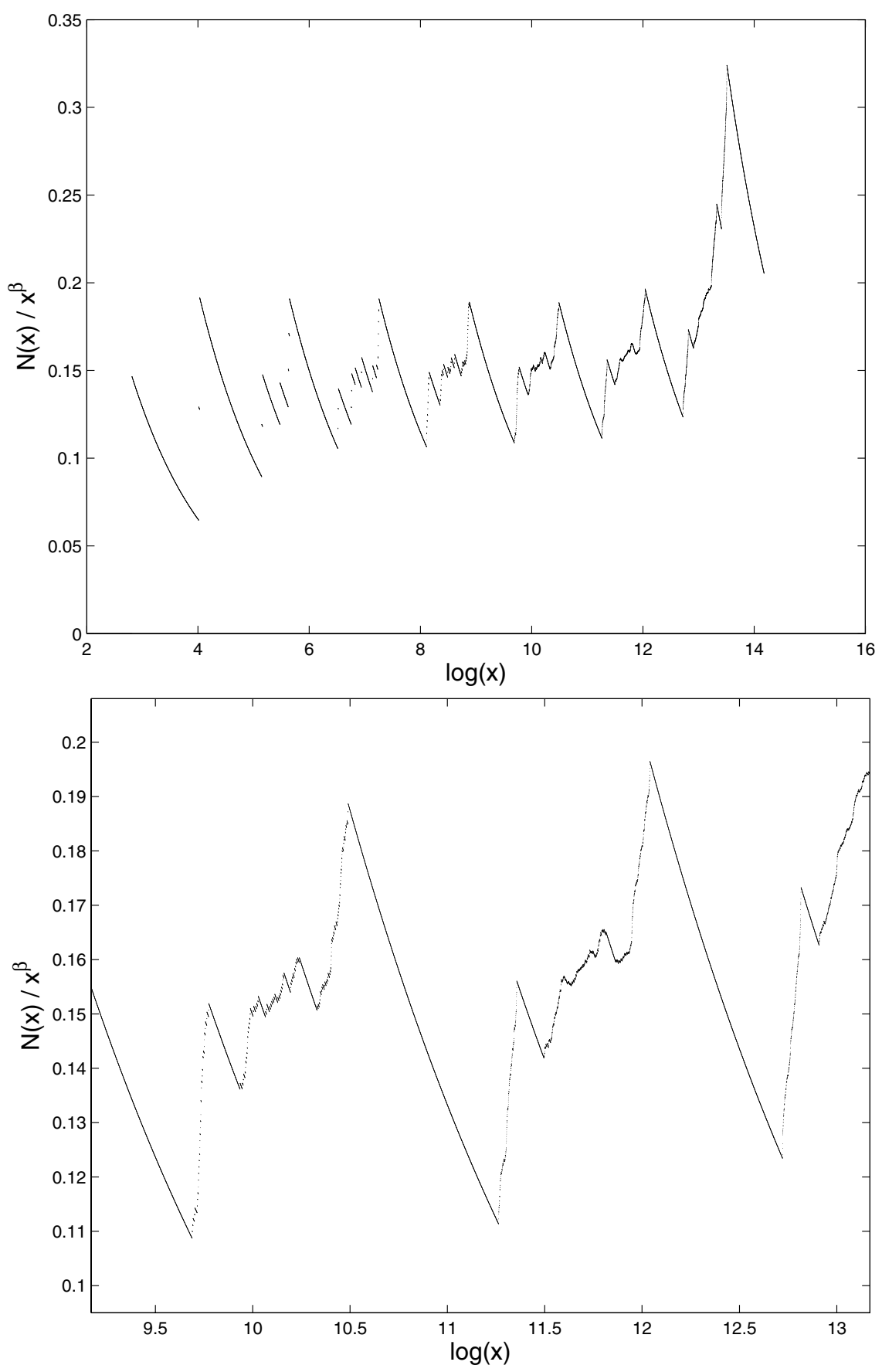

Figure 9. The Weyl ratio for the spectral counting function of $\Delta_{\varphi^{\prime}}$ with $\varphi^{\prime}=\varphi \circ \sigma$ such that $c_{1} \leq \varphi \leq c_{2}, \frac{c_{2}}{c_{1}}=1.1$, and $\sigma$ permutes the level 3 cells of $S G$, as a function of $\log x$ 

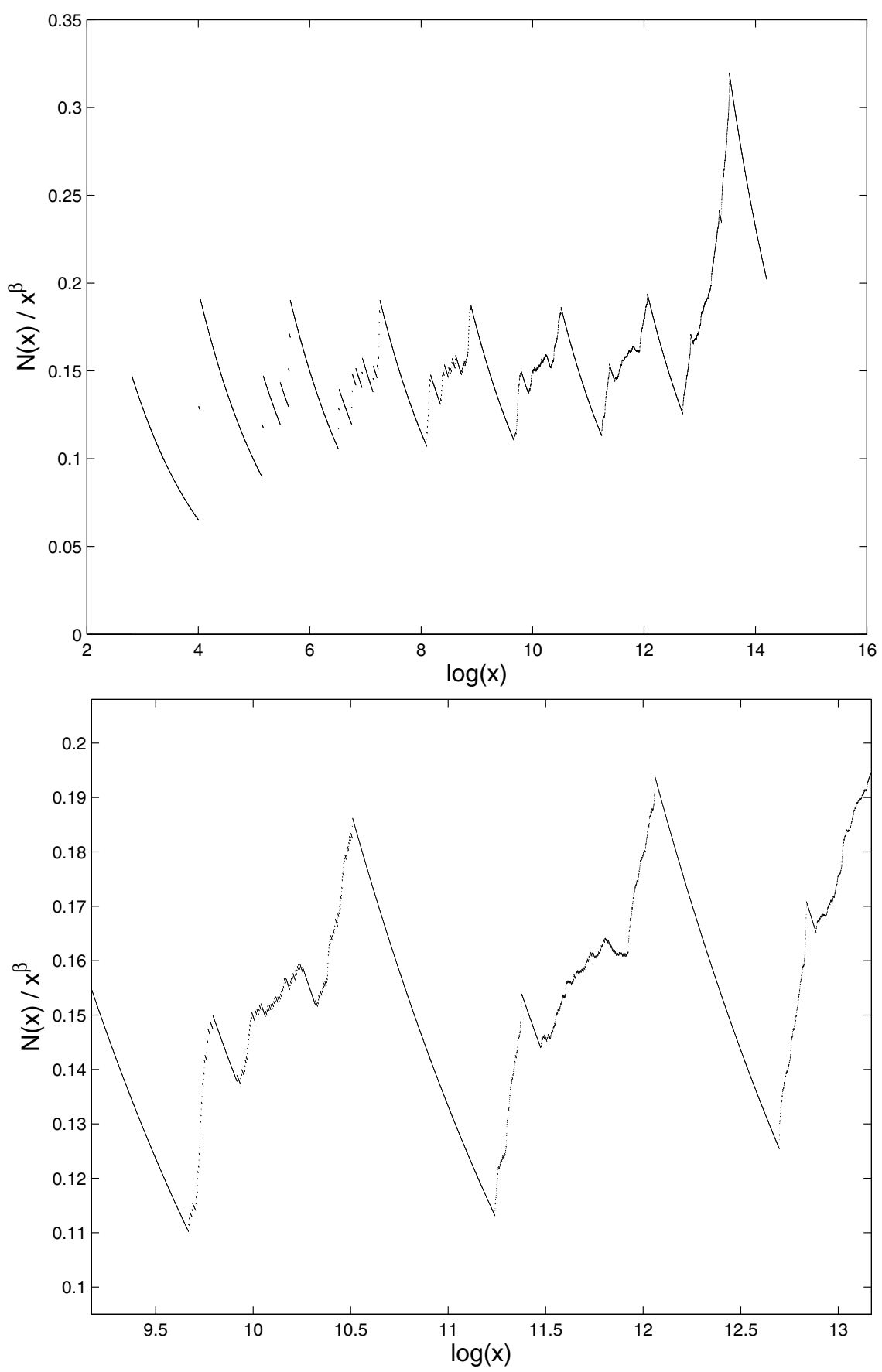

FIgURE 10. The Weyl ratio for the spectral counting function of $\Delta_{\varphi^{\prime}}$ with $\varphi^{\prime}=\varphi \circ \sigma$ such that $c_{1} \leq \varphi \leq c_{2}, \frac{c_{2}}{c_{1}}=1.15$, and $\sigma$ permutes the level 3 cells of $S G$, as a function of $\log x$ 

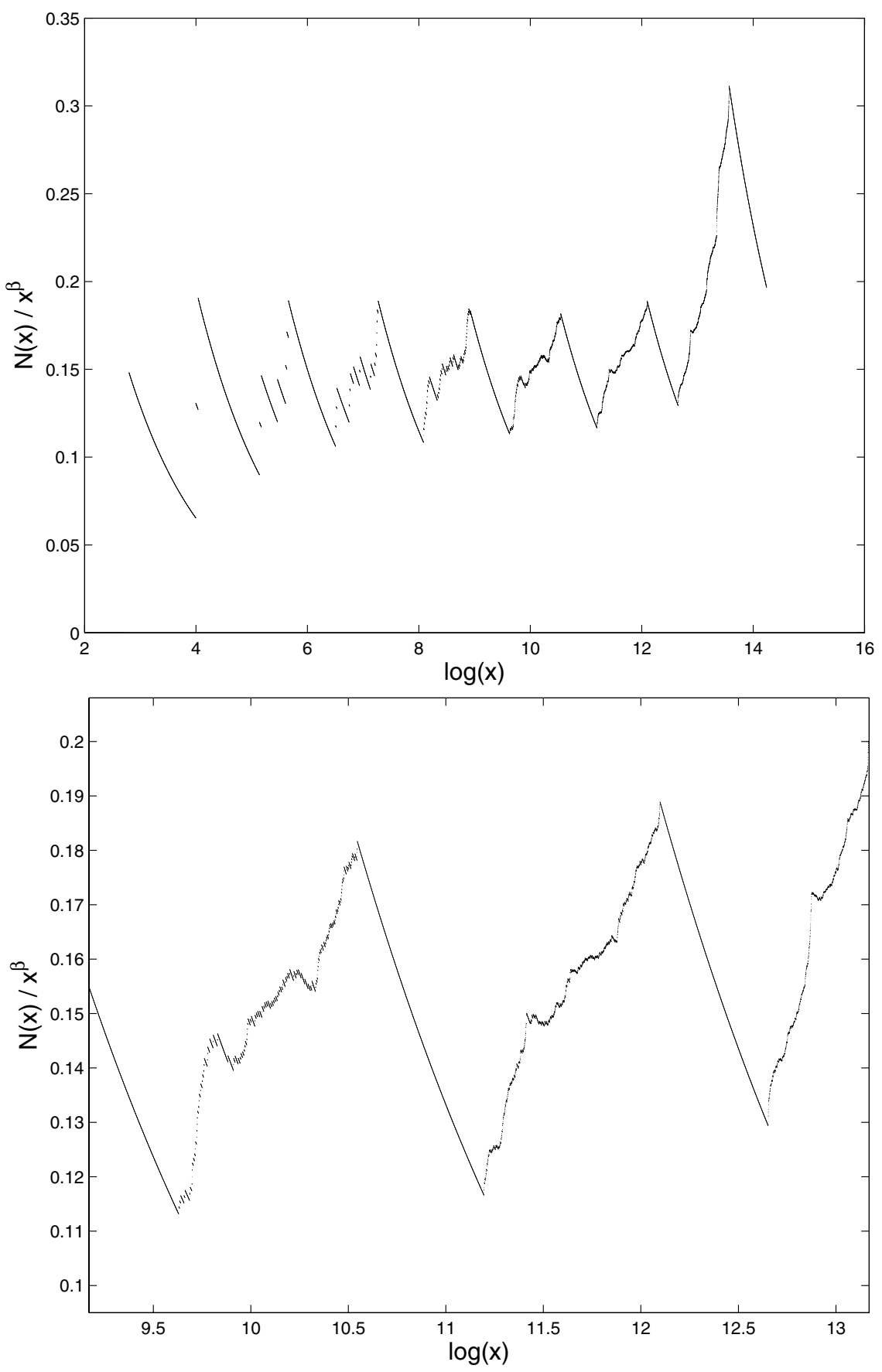

FIgURE 11. The Weyl ratio for the spectral counting function of $\Delta_{\varphi^{\prime}}$ with $\varphi^{\prime}=\varphi \circ \sigma$ such that $c_{1} \leq \varphi \leq c_{2}, \frac{c_{2}}{c_{1}}=1.25$, and $\sigma$ permutes the level 3 cells of $S G$, as a function of $\log x$ 


\section{EnERGy Distribution For HARMONiC FUnCtions ON $S G$}

The objective of this section is to introduce an interesting characterization of energy measures on the Sierpinski Gasket analogous to self-similar measures. We demonstrate the construction in the next theorem (showing some applications afterwards and in the next section) and will show how to replicate it for similar fractals in section 6 .

Theorem 4.1. Let

$$
\begin{gathered}
E_{0}=\frac{1}{75}\left[\begin{array}{ccc}
47 & -3 & -3 \\
14 & 9 & -6 \\
14 & -6 & 9
\end{array}\right], \quad E_{1}=\frac{1}{75}\left[\begin{array}{ccc}
9 & 14 & -6 \\
-3 & 47 & -3 \\
-6 & 14 & 9
\end{array}\right], \\
E_{2}=\frac{1}{75}\left[\begin{array}{ccc}
9 & -6 & 14 \\
-6 & 9 & 14 \\
-3 & -3 & 47
\end{array}\right] .
\end{gathered}
$$

Then, for any harmonic function $h$,

$$
\nu_{h}\left(F_{w} K\right)=\left(\begin{array}{lll}
1 & 1 & 1
\end{array}\right) E_{w_{m}} E_{w_{m-1}} \cdots E_{w_{1}}\left(\begin{array}{c}
\nu_{h}\left(F_{0} K\right) \\
\nu_{h}\left(F_{1} K\right) \\
\nu_{h}\left(F_{2} K\right)
\end{array}\right)
$$

for all words $w=w_{1} w_{2} \ldots w_{m}$.

Proof. We show here that the matrices above are the unique matrices such that $E_{j}\left(\nu_{h}\left(F_{i} K\right)\right)_{i=0}^{2}=\left(\nu_{h}\left(F_{j} F_{i} K\right)\right)_{i=0}^{2}$ for $j=0,1,2$, and (4.1) can be derived recursively. Suppose we are given a harmonic function $h$ defined on the boundary points of $S G$ by $x, y, z$. Subtract off a constant function such that we get the harmonic function with boundary points $0, a, b$, so we assume that $h$ is of this form. Since we subtracted a constant function, the energy of this function (and hence the measure) on the subcells will be the same. Calculating the measure on each cell $F_{0} K, F_{1} K$, and $F_{2} K$, we see that

$$
\begin{aligned}
& \nu_{h}\left(F_{0} K\right)=\frac{2}{5} a^{2}+\frac{2}{5} b^{2}+\frac{2}{5} a b, \\
& \nu_{h}\left(F_{1} K\right)=\frac{6}{5} a^{2}+\frac{2}{5} b^{2}-\frac{6}{5} a b,
\end{aligned}
$$

and

$$
\nu_{h}\left(F_{2} K\right)=\frac{2}{5} a^{2}+\frac{6}{5} b^{2}-\frac{6}{5} a b .
$$

Note that these form a linearly independent set of polynomials. Looking at the measure on the cells $F_{0} F_{0} K, F_{1} F_{0} K, F_{2} F_{0} K$, we would like a linear transformation that will express these in terms of the measures $\nu_{h}\left(F_{0} K\right), \nu_{h}\left(F_{1} K\right)$, and $\nu_{h}\left(F_{2} K\right)$. We can use the equations above to find the measure of the subcells of $F_{0} K$ since that cell is of the form $0, a, b$, but with values $0, \frac{2}{5} a+\frac{1}{5} b, \frac{1}{5} a+\frac{2}{5} b$; hence we can plug in these values for $a$ and $b$ in the above equations to get

and

$$
\begin{aligned}
& \nu_{h}\left(F_{00} K\right)=\frac{14}{75} a^{2}+\frac{14}{75} b^{2}+\frac{26}{75} a b, \\
& \nu_{h}\left(F_{01} K\right)=\frac{14}{75} a^{2}+\frac{2}{75} b^{2}+\frac{2}{75} a b,
\end{aligned}
$$

$$
\nu_{h}\left(F_{02} K\right)=\frac{2}{75} a^{2}+\frac{14}{75} b^{2}+\frac{2}{75} a b .
$$


What we are looking for is a matrix $E_{0}$ such that

$$
E_{0}\left(\begin{array}{c}
\nu_{h}\left(F_{0} K\right) \\
\nu_{h}\left(F_{1} K\right) \\
\nu_{h}\left(F_{2} K\right)
\end{array}\right)=\left(\begin{array}{c}
\nu_{h}\left(F_{00} K\right) \\
\nu_{h}\left(F_{01} K\right) \\
\nu_{h}\left(F_{02} K\right)
\end{array}\right) .
$$

Since each measure is a polynomial in terms of $a$ and $b$, our requirements for the matrix is that the coefficients match up; for example, if $\left(E_{0}\right)_{i j}=e_{i j}$, we want

$$
\begin{aligned}
e_{11}\left(\frac{2}{5} a^{2}+\frac{2}{5} b^{2}+\frac{2}{5} a b\right)+e_{12}\left(\frac{6}{5} a^{2}+\frac{2}{5} b^{2}-\frac{6}{5} a b\right)+ & e_{13}\left(\frac{2}{5} a^{2}+\frac{6}{5} b^{2}-\frac{6}{5} a b\right) \\
& =\frac{14}{75} a^{2}+\frac{14}{75} b^{2}+\frac{26}{75} a b .
\end{aligned}
$$

From this, and the fact that the coefficients of this linear combination of polynomials must match the coefficients of the polynomial on the right, we get a set of linear equations

and

$$
\begin{aligned}
& \frac{2}{5} e_{11}+\frac{6}{5} e_{12}+\frac{2}{5} e_{13}=\frac{14}{75} \\
& \frac{2}{5} e_{11}+\frac{2}{5} e_{12}+\frac{6}{5} e_{13}=\frac{14}{75}
\end{aligned}
$$

$$
\frac{2}{5} e_{11}-\frac{6}{5} e_{12}-\frac{6}{5} e_{13}=\frac{26}{75}
$$

corresponding to the matching conditions for the coefficients of $a^{2}, b^{2}$, and $a b$ respectively. Solving this system, we find that ${ }^{1} e_{11}=\frac{47}{75}, e_{12}=-\frac{3}{75}$, and $e_{13}=-\frac{3}{75}$. Doing the same calculations for the other rows of $E_{0}$ we find that

$$
E_{0}=\frac{1}{75}\left[\begin{array}{ccc}
47 & -3 & -3 \\
14 & 9 & -6 \\
14 & -6 & 9
\end{array}\right]
$$

To find the matrices $E_{1}$ and $E_{2}$ that take the level one measures to the level two measures in $F_{1} K$ and $F_{2} K$ respectively, we rotate $S G$ and note that, if a harmonic function $h$ has measures $x, y, z$ on the cells $F_{0} K, F_{1} K, F_{2} K$ respectively and if $x^{\prime}, y^{\prime}, z^{\prime}$ are the level two measures on $F_{1} K$, then

$$
E_{0}\left(\begin{array}{l}
y \\
z \\
x
\end{array}\right)=\left(\begin{array}{l}
y^{\prime} \\
z^{\prime} \\
x^{\prime}
\end{array}\right)
$$

or equivalently,

$$
\left(\begin{array}{l}
x^{\prime} \\
y^{\prime} \\
z^{\prime}
\end{array}\right)=\left[\begin{array}{lll}
0 & 0 & 1 \\
1 & 0 & 0 \\
0 & 1 & 0
\end{array}\right] E_{0}\left[\begin{array}{lll}
0 & 1 & 0 \\
0 & 0 & 1 \\
1 & 0 & 0
\end{array}\right]\left(\begin{array}{l}
x \\
y \\
z
\end{array}\right)
$$

Therefore,

$$
E_{1}=\frac{1}{75}\left[\begin{array}{ccc}
9 & 14 & -6 \\
-3 & 47 & -3 \\
-6 & 14 & 9
\end{array}\right]
$$

\footnotetext{
${ }^{1}$ Equivalently, if we let $A$ be the matrix whose rows are the coefficients of $a^{2}, b^{2}$, and $a b$ for each $\nu_{h}\left(F_{i} K\right)$ and define $B$ similarly for $\nu_{h}\left(F_{0 i} K\right)$, then $E_{0}=B A^{-1}$.
} 
A similar computation shows that

$$
E_{2}=\frac{1}{75}\left[\begin{array}{ccc}
9 & -6 & 14 \\
-6 & 9 & 14 \\
-3 & -3 & 47
\end{array}\right]
$$

When using these matrices, however, the question arises of what are the possible combinations of first level measures. Clearly, we cannot have that $\nu_{h}\left(F_{0} K\right)=$ $\nu_{h}\left(F_{1} K\right)=\nu_{h}\left(F_{2} K\right)=1$.

Theorem 4.2. The domain of admissible measures is a circular cone in $\mathbb{R}^{3}$ defined by the relation

$$
x^{2}+y^{2}+z^{2}=\frac{11}{25}(x+y+z)^{2}
$$

where $x, y$, and $z$ are the first level measures $\nu_{h}\left(F_{0} K\right), \nu_{h}\left(F_{1} K\right)$ and $\nu_{h}\left(F_{2} K\right)$ respectively.

Proof. Setting $x, y$, and $z$ equal to the equations for the measures in equations (4.2) through (4.4), we use some linear algebra to solve for $a^{2}, b^{2}$, and $a b$ and get

$$
\begin{aligned}
a^{2} & =\frac{3}{4} x+\frac{3}{4} y-\frac{1}{2} z, \\
b^{2} & =\frac{3}{4} x-\frac{1}{2} y+\frac{3}{4} z, \\
a b & =x-\frac{1}{4} y-\frac{1}{4} z .
\end{aligned}
$$

We use these expressions by solving the expression $a^{2} b^{2}=(a b)^{2}$ and, after some algebra, we get

Then

$$
x y+x z+y z=\frac{7}{11}\left(x^{2}+y^{2}+z^{2}\right) .
$$

$$
(x+y+z)^{2}=x^{2}+y^{2}+z^{2}+2(x y+x z+y z)=\frac{25}{11}\left(x^{2}+y^{2}+z^{2}\right),
$$

which then gives us (4.6). This defines a circular cone in $\mathbb{R}^{3}$ of admissible measures on the Sierpinski Gasket. If a vector of measures satisfies the above equation, then the corresponding class of harmonic functions can be solved for by the expressions for $a^{2}$ and $b^{2}$.

Using Theorem 4.1, we get the following theorem:

Theorem 4.3. Let $m \in \mathbb{N}$. Then for all harmonic functions $h$,

$$
\max _{|w|=m} \nu_{h}\left(F_{w} K\right)=\max _{i=0,1,2} \nu_{h}\left(F_{i}^{m} K\right),
$$

where the level $m$ cell of maximum measure will be contained in the level 1 cell of maximum measure.

This theorem is analogous to the classical result that the norm of the gradient of a harmonic function achieves its maximum on the boundary, which follows from the fact that, in the classical case, for a harmonic function $h,\|\nabla h\|^{2}$ is a subharmonic function. Here we can consider $\nu_{h}\left(F_{w} K\right)$ to be analogous to $\int_{F_{w} K}\|\nabla h\|^{2}$. For other notions of gradients on fractals, see [11], [12], [7], [24], and [23]. 
Proof. Let $x, y, z$ be the first level measures of $F_{0} K, F_{1} K$, and $F_{2} K$ respectively and, without loss of generality, assume $x \geq y, z$. The case $m=1$ is trivial. By induction, assume the hypothesis is true for all integers less than some $m \in \mathbb{N}$. By hypothesis, if we restrict our focus to the subcells $F_{i} K$, we know that the maximum of the measures of the level $m-1$ cells with respect to these cells (which are level $m$ cells of all of $S G$ ) must occur on one of the boundary cells $F_{i} F_{j}^{m-1} K$ for $i, j=0,1,2$, and hence we know that

$$
\max _{|w|=m} \nu_{h}\left(F_{w} K\right)=\max _{i, j=0,1,2} \nu_{h}\left(F_{i} F_{j}^{m-1} K\right) .
$$

Using the matrix $E_{0}$, it is easy to show that $\nu_{h}\left(F_{0}^{2} K\right)=\max _{j=0,1,2} \nu_{h}\left(F_{0} F_{j} K\right)$, so by hypothesis we know that $\max _{j=0,1,2} \nu_{h}\left(F_{0} F_{j}^{m-1}\right)=\nu_{h}\left(F_{0}^{m} K\right)$, so we have eliminated the $i=0$ case from the above equation. Let $F_{w}=F_{1} F_{0}^{m-1}, v=(1,1,1)$ and $e=(x, y, z)^{T}$. Note that, by diagonalization, if $D=\operatorname{diag}\{\alpha, \beta, \gamma\}$ where $\alpha=\frac{1}{5}$, $\beta=\frac{1}{15}$, and $\gamma=\frac{3}{5}$ are the eigenvalues of the $E_{0}$ and $P=\left[\begin{array}{ccc}0 & 1 & 3 \\ -1 & 7 & 1 \\ 1 & 7 & 1\end{array}\right]$ is the matrix of eigenvectors of $E_{0}$, then

$$
\begin{aligned}
v E_{0}^{m} & =v P D^{m} P^{-1} \\
& =v \frac{1}{40}\left[\begin{array}{ccc}
-2 \beta^{m}+42 \gamma^{m} & 3 \beta^{m}-3 \gamma^{m} & 3 \beta^{m}-3 \gamma^{m} \\
-14 \beta^{m}+14 \gamma^{m} & 20 \alpha^{m}+21 \beta^{m}-\gamma^{m} & -20 \alpha^{m}+21 \beta^{m}-\gamma^{m} \\
-14 \beta^{m}+14 \gamma^{m} & -20 \alpha^{m}+21 \beta^{m}-\gamma^{m} & 20 \alpha^{m}+21 \beta^{m}-\gamma^{m}
\end{array}\right] \\
& =\frac{1}{8}\left(\begin{array}{c}
-6 \beta^{m}+14 \gamma^{m} \\
9 \beta^{m}-\gamma^{m} \\
9 \beta^{m}-\gamma^{m}
\end{array}\right)^{T} .
\end{aligned}
$$

So

$$
\begin{aligned}
& \nu_{h}\left(F_{0}^{m} K\right)-\nu_{h}\left(F_{w}\right) \\
& =v\left(E_{0}^{m}-E_{0}^{m-1} E_{1}\right) e=v\left(P D^{m} P^{-1}-P D^{m-1} P^{-1} E_{1}\right) e \\
& =\frac{1}{8}\left[\left(\begin{array}{c}
-6 \beta^{m}+14 \gamma^{m} \\
9 \beta^{m}-\gamma^{m} \\
9 \beta^{m}-\gamma^{m}
\end{array}\right)^{T}-\left(\begin{array}{c}
-6 \beta^{m-1}+14 \gamma^{m-1} \\
9 \beta^{m-1}-\gamma^{m-1} \\
9 \beta^{m-1}-\gamma^{m-1}
\end{array}\right)^{T} E_{1}\right] e \\
& =\frac{1}{8}\left[\left(\begin{array}{c}
-6 \beta^{m}+14 \gamma^{m} \\
9 \beta^{m}-\gamma^{m} \\
9 \beta^{m}-\gamma^{m}
\end{array}\right)-\frac{1}{5}\left(\begin{array}{c}
-9 \beta^{m-1}+9 \gamma^{m-1} \\
31 \beta^{m-1}+9 \gamma^{m-1} \\
6 \beta^{m-1}-6 \gamma^{m-1}
\end{array}\right)^{T}\right] e \\
& =x\left(\frac{21}{8} \beta^{m}+\frac{11}{8} \gamma^{m}\right)-y\left(\frac{21}{2} \beta^{m}-\frac{1}{2} \gamma^{m}\right)+z\left(-\frac{9}{8} \beta^{m}+\frac{1}{8} \gamma^{m}\right) .
\end{aligned}
$$

Since we're assuming $x \geq y, z$, it follows that

$$
\nu_{h}\left(F_{0}^{m} K\right)-\nu_{h}\left(F_{w}\right) \geq\left(\frac{11}{8} \gamma^{m}-9 \beta^{m}\right) x,
$$

which is greater than zero for $m>1$. By symmetry, we know that this is also a lower bound for $\nu_{h}\left(F_{0}^{m} K\right)-\nu_{h}\left(F_{2} F_{0}^{m}\right)$. 
Now consider the cell $F_{2} F_{1}^{m-1}$. Let $Q=\left[\begin{array}{lll}0 & 0 & 1 \\ 1 & 0 & 0 \\ 0 & 1 & 0\end{array}\right]$. By (4.5),

$$
\begin{aligned}
\nu_{h}\left(F_{0}^{m} K\right) & -\nu_{h}\left(F_{2} F_{1}^{m-1}\right)=v E_{0}^{m} e-v E_{1}^{m-1} E_{2} e=v E_{0}^{m} e-v Q E_{0}^{m-1} Q^{-1} E_{2} e \\
& =v E_{0}^{m} e-\left(v E_{0}^{m-1}\right) Q^{-1} E_{2} \\
& =\frac{1}{8}\left(\begin{array}{c}
-6 \beta^{m}+14 \gamma^{m} \\
9 \beta^{m}-\gamma^{m} \\
9 \beta^{m}-\gamma^{m}
\end{array}\right) e-\frac{1}{8}\left(\begin{array}{c}
-6 \beta^{m-1}+14 \gamma^{m-1} \\
9 \beta^{m-1}-\gamma^{m-1} \\
9 \beta^{m-1}-\gamma^{m-1}
\end{array}\right){ }^{T} Q^{-1} E_{2} e \\
& =\frac{1}{8}\left[\left(\begin{array}{c}
-6 \beta^{m}+14 \gamma^{m} \\
9 \beta^{m}-\gamma^{m} \\
9 \beta^{m}-\gamma^{m}
\end{array}\right)-\left(\begin{array}{c}
9 \beta^{m-1}-\gamma^{m-1} \\
-6 \beta^{m-1}+14 \gamma^{m-1} \\
9 \beta^{m-1}-\gamma^{m-1}
\end{array}\right) E_{2}^{T}\right] e \\
& =\frac{1}{8}\left[\left(\begin{array}{c}
-6 \beta^{m}+14 \gamma^{m} \\
9 \beta^{m}-\gamma^{m} \\
9 \beta^{m}-\gamma^{m}
\end{array}\right)-\frac{1}{5}\left(\begin{array}{c}
6 \beta^{m-1}=6 \gamma^{m-1} \\
-9 \beta^{m-1}+9 \gamma^{m-1} \\
31 \beta^{m-1}+9 \gamma^{m-1}
\end{array}\right)\right]^{T} e \\
= & x\left(-3 \beta^{m}+2 \gamma^{m}\right)+y\left(\frac{9}{2} \beta^{m}-\frac{1}{2} \gamma^{m}\right)+z\left(-\frac{21}{2} \beta^{m}-\frac{1}{2} \gamma^{m}\right),
\end{aligned}
$$

and since we are assuming $x \geq y, z$, we therefore know that

$$
\nu_{h}\left(F_{0}^{m} K\right)-\nu_{h}\left(F_{2} F_{1}^{m-1} K\right) \geq \gamma^{m}-\frac{27}{2} \beta^{m},
$$

which is greater than zero for $m>1$. By symmetry, we also know that this is a lower bound for $\nu_{h}\left(F_{0}^{m} K\right)-\nu_{h}\left(F_{1} F_{2}^{m-1} K\right)$. Therefore, $\nu_{h}\left(F_{0}^{m}\right)$ is the maximum of all level $m$ cells.

We prove a corollary of this result concerning $L^{p}$ dimensions at the end of the next section.

\section{5. $L^{p}$ DIMENSIONS ON ENERGY MEASURES}

Having looked at the admissible measures and these matrices, we now have methods of computing the $L^{p}$ dimensions of energy measures on $S G$ for integer values of $p$, where

$$
\operatorname{dim}_{p} \nu_{h}=\lim _{m \rightarrow \infty} \frac{\log \sum_{|w|=m} \nu_{h}\left(F_{w} K\right)^{p}}{(p-1) m \log \frac{3}{5}}
$$

as defined in [17].

Theorem 5.1. For all harmonic functions $h$, $\operatorname{dim}_{2} \nu_{h}=\frac{\log \frac{25}{11}}{\log \frac{5}{3}} \approx 1.6071639985 \ldots$.

Proof. Let $w$ be any word of finite length. By Theorem 4.2,

$$
\nu_{h}\left(F_{w 0} K\right)^{2}+\nu_{h}\left(F_{w 1} K\right)^{2}+\nu_{h}\left(F_{w 2} K\right)^{2}=\frac{11}{25} \nu_{h}\left(F_{w}\right)^{2} .
$$

By induction, we see that

$$
\sum_{|w|=m} \nu_{h}\left(F_{w} K\right)=\left(\frac{11}{25}\right)^{m} \nu_{h}(K) .
$$


Substituting this into (5.1) for $p=2$, we get

$$
\operatorname{dim}_{2} \nu_{h}=\lim _{m \rightarrow \infty} \frac{\log \left(\left(\frac{11}{25}\right)^{m} \nu_{h}(K)\right)}{m \log \frac{3}{5}}=\frac{\log \frac{25}{11}}{\log \frac{5}{3}} .
$$

Note that this does not depend on our choice of harmonic function.

Theorem 5.2. For all harmonic functions $h$,

$$
\operatorname{dim}_{3} \nu_{h}=\frac{\log \left(\frac{31}{225}+\frac{151730163445790^{1 / 2}}{134217728}\right)}{2 \log \frac{3}{5}} \approx 1.4404335708 \ldots
$$

Proof. Here it's a little trickier. Let $x, y$, and $z$ denote $\nu_{h}\left(F_{w 0} K\right), \nu_{h}\left(F_{w 1} K\right)$, and $\nu_{h}\left(F_{w 2} K\right)$ respectively for some word $w$. We use equation (5.1) to get

$$
\begin{aligned}
(x+y+z)^{3} & =x^{3}+y^{3}+z^{3}+3\left(x^{2} y+x^{2} z+y^{2} x+y^{2} z+z^{2} x+z^{2} y\right)+6 x y z \\
& =-2\left(x^{3}+y^{3}+z^{3}\right)+3\left(x^{2}+y^{2}+z^{2}\right)(x+y+z)+6 x y z \\
& =-2\left(x^{3}+y^{3}+z^{3}\right)+\frac{33}{25}(x+y+z)^{3}+6 x y z,
\end{aligned}
$$

and thus

$$
x^{3}+y^{3}+z^{3}=\frac{4}{25}(x+y+z)^{3}+3 x y z .
$$

We need a recurrence relation on $x y z$ for this to work. Consider the second level cell measures $x_{1}, x_{2}, x_{3}, y_{1}, y_{2}, y_{3}, z_{1}, z_{2}, z_{3}$ of a cell $F_{w} K$, where the $x_{i}, y_{i}, z_{i}$ are the measures of the subcells of $F_{w} F_{0}, F_{w} F_{1}$, and $F_{w} F_{2}$ respectively. By the matrices $E_{i}$, we can substitute linear expressions for $x_{i}, y_{i}$, and $z_{i}$ in terms of $x, y, z$. Doing this, we get

$$
\begin{aligned}
& x_{1} x_{2} x_{3}+y_{1} y_{2} y_{3}+z_{1} z_{2} z_{3} \\
& =\frac{1}{3^{3} 5^{6}}\left[9536\left(x^{3}+y^{3}+z^{3}\right)-1467\left(x^{2} y+x^{2} z+y^{2} x+y^{2} z+z^{2} x+z^{2} y\right)\right. \\
& +15741 x y z] \\
& =\frac{1}{3^{3} 5^{6}}\left[(9536+1467)\left(x^{3}+y^{3}+z^{3}\right)-1467\left(x^{2}+y^{2}+z^{2}\right)(x+y+z)+15741 x y z\right] \\
& =\frac{1}{3^{3} 5^{6}}\left[11003\left(\frac{4}{25}(x+y+z)^{3}+3 x y z\right)-1467\left(\frac{11}{25}(x+y+z)^{2}\right)(x+y+z)\right. \\
& =\frac{223}{3^{3} 5^{5}}(x+y+z)^{3}+\frac{26}{3^{2} 5^{2}} x y z .
\end{aligned}
$$

Therefore, we have a recurrence relation on the sums of cubes of level two subcells and the sums of the products of level two subcells. Suppose we have

$$
\sum_{i=0}^{2}\left[a\left(\sum_{j=0}^{2} \nu_{h}\left(F_{w i j} K\right)\right)^{3}+b \nu_{h}\left(F_{w i 0} K\right) \nu_{h}\left(F_{w i 1} K\right) \nu_{h}\left(F_{w i 2} K\right)\right] \text {. }
$$


Then this is equal to

$$
a^{\prime}\left(\left(\nu_{h}\left(F_{w 0} K\right)+\nu_{h}\left(F_{w 1} K\right)+\nu_{h}\left(F_{w 2} K\right)\right)^{3}+b^{\prime} \nu_{h}\left(F_{w 0} K\right) \nu_{h}\left(F_{w 1} K\right) \nu_{h}\left(F_{w 2} K\right)\right.
$$

where

$$
\left(\begin{array}{l}
a^{\prime} \\
b^{\prime}
\end{array}\right)=\underbrace{\left[\begin{array}{cc}
\frac{4}{25} & \frac{223}{3^{3} \cdot 5^{5}} \\
3 & \frac{26}{3^{2} \cdot 5^{2}}
\end{array}\right]}_{M_{3}}\left(\begin{array}{l}
a \\
b
\end{array}\right)
$$

By induction, we have that

$$
\sum_{|w|=m} \nu_{h}\left(F_{w} K\right)^{3}=\left(\begin{array}{c}
\nu_{h}(K)^{3} \\
\nu_{h}\left(F_{0} K\right) \nu_{h}\left(F_{1} K\right) \nu_{h}\left(F_{2} K\right)
\end{array}\right)^{T} M_{3}^{m-1}\left(\begin{array}{l}
1 \\
0
\end{array}\right) .
$$

Therefore, when taking the limit of these sums in the log, the limit of the log divided by $m$ should equal the log of the maximum eigenvalue; hence

$$
\operatorname{dim}_{3} \nu_{h}=\frac{\log \left(\frac{31}{225}+\frac{151730163445790^{1 / 2}}{134217728}\right)}{2 \log \frac{3}{5}} .
$$

Theorem 5.3. For all harmonic functions $h$,

$$
\operatorname{dim}_{4} \nu_{h}=\frac{\log \left(\frac{1327}{16875}+\frac{3242319174104421^{1 / 2}}{1073741824}\right)}{3 \log \frac{3}{5}} \approx 1.3230040245 \ldots
$$

Proof. This time we will have an $(x+y+z) x y z$ term in our recurrence relation instead of an $x y z$ term:

$$
\begin{aligned}
(x+y+z)^{4}= & x^{4}+y^{4}+z^{4}+4\left(x^{3} y+x^{3} z+y^{3} x+y^{3} z+z^{3} x+z^{3} y\right) \\
& +6\left(x^{2} y^{2}+x^{2} z^{2}+y^{2} z^{2}\right)+12(x+y+z) x y z \\
=- & 6\left(x^{4}+y^{4}+z^{4}\right)+4\left(x^{3}+y^{3}+z^{3}\right)(x+y+z)+3\left(x^{2}+y^{2}+z^{2}\right)^{2} \\
& \quad+12(x+y+z) x y z \\
= & -6\left(x^{4}+y^{4}+z^{4}\right)+\left(\frac{16}{25}+\frac{363}{625}\right)(x+y+z)^{4}+(12+12)(x+y+z) x y z \\
= & -6\left(x^{4}+y^{4}+z^{4}\right)+\frac{763}{625}(x+y+z)^{4}+24(x+y+z) x y z .
\end{aligned}
$$

Hence,

$$
x^{4}+y^{4}+z^{4}=\frac{23}{625}(x+y+z)^{4}+4 x y z .
$$

To find the recurrence relation for $(x+y+z) x y z$, we again look at

$$
\left(x_{1}+x_{2}+x_{3}\right) x_{1} x_{2} x_{3}+\left(y_{1}+y_{2}+y_{3}\right) y_{1} y_{2} y_{3}+\left(z_{1}+z_{2}+z_{3}\right) z_{1} z_{2} z_{3}
$$

as measures of subcells of three subcells with measures $x, y, z$ respectively. Describing them as linear combinations of the first level measures $x, y, z$ with the matrices 
$E_{i}$, we find that the above equation is equal to

$$
\begin{aligned}
& \frac{1}{3^{4} 5^{6}}\left(27636\left(x^{4}+y^{4}+z^{4}\right)+4644\left(x^{3} y+x^{3} z+y^{3} x+y^{3} z+z^{3} x+z^{3} y\right)\right. \\
& \left.-15984\left(x^{2} y^{2}+x^{2} z^{2}+y^{2} z^{2}\right)+14607(x+y+z) x y z\right) \\
& =\frac{1}{3^{4} 5^{6}}\left((27636-4644+7992)\left(x^{4}+y^{4}+z^{4}\right)+4644\left(x^{3}+y^{3}+z^{3}\right)(x+y+z)\right. \\
& \left.-7992\left(x^{2}+y^{2}+z^{2}\right)^{2}+14607(x+y+z) x y z\right) \\
& =\frac{1}{3^{4} 5^{6}}\left(30984\left(\frac{23}{625}(x+y+z)^{4}+4(x+y+z) x y z\right)+4644 \frac{4}{25}(x+y+z)^{3}\right. \\
& \left.+3 x y z)(x+y+z)-7992\left(\frac{11}{25}(x+y+z)^{2}\right)^{2}+14607(x+y+z) x y z\right) \\
& =\frac{1}{3^{4} 5^{6}}\left(336(x+y+z)^{4}+152475(x+y+z) x y z\right)=\frac{112}{3^{3} 5^{6}}(x+y+z)^{4} \\
& +\frac{2033}{3^{3} 5^{4}}(x+y+z) x y z \text {. }
\end{aligned}
$$

Therefore, the recurrence relation on the measure is modeled by

$$
\begin{aligned}
& \sum_{|w|=m} \nu_{h}\left(F_{w} K\right)^{4} \\
& =\left(\begin{array}{c}
\nu_{h}(K)^{4} \\
\left(\nu_{h}\left(F_{0} K\right)+\nu_{h}\left(F_{1} K\right)+\nu_{h}\left(F_{2} K\right)\right) \nu_{h}\left(F_{0} K\right) \nu_{h}\left(F_{1} K\right) \nu_{h}\left(F_{2} K\right)
\end{array}\right)^{T} M_{4}^{m-1}\left(\begin{array}{c}
1 \\
0
\end{array}\right)
\end{aligned}
$$

where

$$
M_{4}=\left[\begin{array}{cc}
\frac{23}{625} & \frac{112}{33^{6}} \\
4 & \frac{2033}{3^{3} 5^{4}}
\end{array}\right]
$$

Thus, using the maximum eigenvalue of $M_{4}$ in the log, we obtain

$$
\operatorname{dim}_{4} \nu_{h}=\frac{\log \left(\frac{1327}{16875}+\frac{3242319174104421^{1 / 2}}{1073741824}\right)}{3 \log \frac{3}{5}} .
$$

For larger $p$, the calculations get more and more arduous. In fact, we have the following lemma and theorem:

Lemma 5.4. Let $x, y, z$ be the first level measures of $S G$. For $p \in \mathbb{N}$, there exist coefficients $\left(a_{k}^{(p)}\right)_{k=0}^{\left\lfloor\frac{p}{3}\right\rfloor}$ such that

$$
x^{p}+y^{p}+z^{p}=\sum_{k=0}^{\left\lfloor\frac{p}{3}\right\rfloor} a_{k}^{(p)}(x+y+z)^{p-3 k}(x y z)^{k} .
$$

Proof. We prove by induction. Assume the hypothesis holds for the first $p-1$ steps. Let $\alpha=x+y+z$ and $\beta=x y z$. We need to solve for $\left(a_{k}^{(p)}\right)$ in terms of the coefficients $\left(a_{k}^{(n)}\right)$ for $n<p$. Note that

$$
(x+y+z)^{p}=\sum_{i+j+k=p} \frac{p !}{i ! j ! k !} x^{i} y^{j} z^{k},
$$

so define

$$
a_{i j}=\varphi_{j, p-3 i-j} \frac{p !}{i !(i+j) !(p-2 i-j) !}, \text { where } \varphi_{n m}= \begin{cases}1 & \text { if } n \neq m \\ \frac{1}{2} & \text { if } n=m\end{cases}
$$


Define $s(n)=x^{n}+y^{n}+z^{n}$.

Also, recall that the product of two finite power series with coefficients $s=\left(s_{i}\right)$ and $t=\left(t_{i}\right)$ is

$$
\left(\sum_{i=0}^{n} s_{i} x^{i}\right)\left(\sum_{j=0}^{m} t_{j} x^{j}\right)=\sum_{i=0}^{n+m} c_{i}(s, t) x^{i}, \text { where } c_{i}(s, t)=\sum_{k=i-\min \{i, n\}}^{\min \{i, m\}} s_{i-k} t_{k} .
$$

For bookkeeping, define $c_{k}(i, j)=c_{k}\left(a^{(i)}, a^{(p-j)}\right)$. Then

$$
\left.\begin{array}{rl}
\alpha^{p}= & \sum_{i=0}^{\left\lfloor\frac{p}{3}\right\rfloor}(x y z)^{i}\left[\sum_{j=1}^{\left\lfloor\frac{p-3 i}{2}\right\rfloor} a_{i j}\left(x^{j} y^{p-3 i-j}+x^{p-3 i-j} y^{j}+\cdots+y^{j} z^{p-3 i-j}+y^{p-3 i-j} z^{j}\right)\right. \\
+ & \left.+a_{i 0} s(p-3 i)\right]
\end{array}\right]
$$


and, since $\left\lfloor\frac{j}{3}\right\rfloor+\left\lfloor\frac{p-3 i-j}{3}\right\rfloor$ is either $\left\lfloor\frac{p-3 i}{3}\right\rfloor$ or $\left\lfloor\frac{p-3 i}{3}\right\rfloor-1$ for each $i$, if we define $c_{\left\lfloor\frac{p-3 i}{3}\right\rfloor}\left(a^{(j)}, a^{(p-3 i-j)}\right)=0$ if it is the latter case, then this is

$$
\begin{aligned}
& =\sum_{i=1}^{\left\lfloor\frac{p}{3}\right\rfloor}\left[\sum_{j=1}^{\left\lfloor\frac{p-3 i}{2}\right\rfloor} a_{i j} \sum_{k=0}^{\left\lfloor\frac{p-3 i}{3}\right\rfloor} c_{k}(j, 3 i+j) \alpha^{p-3(i+k)} \beta^{i+k}+\sum_{k=0}^{\left\lfloor\frac{p-3 i}{3}\right\rfloor} a_{i} a_{k}^{(p-3 i)} \alpha^{p-3(i+k)} \beta^{i+k}\right] \\
& +\sum_{j=1}^{\left\lfloor\frac{p}{2}\right\rfloor} a_{0 j} \sum_{k=0}^{\left\lfloor\frac{p}{3}\right\rfloor} c_{k}(j, j) \alpha^{p-3 i} \beta^{k}+a_{0} s(p)
\end{aligned}
$$

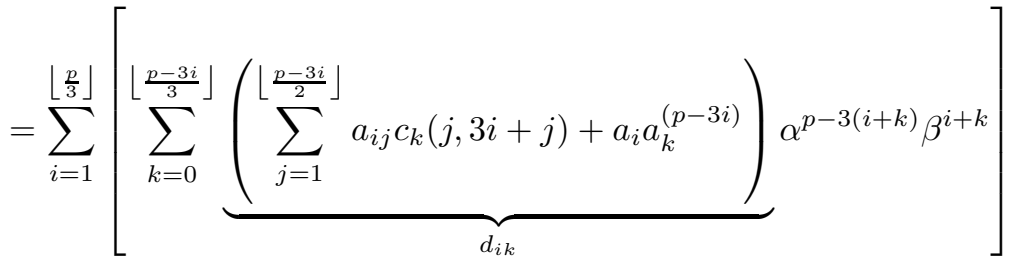

$$
\begin{aligned}
& +\sum_{k=0}^{\left\lfloor\frac{p}{3}\right\rfloor} \underbrace{\left(\sum_{j=1}^{\left\lfloor\frac{p}{2}\right\rfloor} a_{0 j} c_{k}(j, j)\right)}_{e_{k}} \alpha^{p-3 k} \beta^{k}+a_{0} s(p) \\
& =\sum_{i=1}^{\left\lfloor\frac{p}{3}\right\rfloor} \sum_{k=0}^{\left\lfloor\frac{p}{3}\right\rfloor-i} d_{i k} \alpha^{p-3(i+k)} \beta^{i+k}+\sum_{k=0}^{\left\lfloor\frac{p}{3}\right\rfloor} e_{k} \alpha^{p-3 k} \beta^{k}+a_{0} s(p) \\
& =\sum_{k=1}^{\left\lfloor\frac{p}{3}\right\rfloor}\left(\sum_{i=1}^{k} d_{i, k-i}+e_{k}\right) \alpha^{p-3 k} \beta^{k}+e_{0} \alpha^{p}+a_{0} s(p) .
\end{aligned}
$$

Therefore, remembering that this is all equal to $\alpha$, we solve and get $x^{p}+y^{p}+z^{p}=$ $s(p)=\sum_{k=0}^{\left\lfloor\frac{p}{3}\right\rfloor} a_{k}^{(p)} \alpha^{p-3 k} \beta^{k}$, where

$$
\begin{aligned}
a_{k}^{(p)}= & \frac{\sum_{i=1}^{k} d_{i, k-i}+e_{k}}{-a_{0}} \\
= & \frac{\sum_{i=1}^{k}\left[\sum_{j=1}^{\left\lfloor\frac{p-3 i}{2}\right\rfloor} a_{i j} c_{k-i}(j, 3 i+j)+\left(a_{i 0}-\sum_{j=1}^{\left\lfloor\frac{p-3 i}{2}\right\rfloor} a_{i j}\right) a_{k-i}^{(p-3 i)}\right]}{\sum_{j=1}^{\left\lfloor\frac{p}{2}\right\rfloor} a_{0 j}-a_{00}} \\
& \frac{+\sum_{j=1}^{\left\lfloor\frac{p}{2}\right\rfloor} a_{0 j} c_{k}(j, j)}{\sum_{j=1}^{\left\lfloor\frac{p}{2}\right\rfloor} a_{0 j}-a_{00}}
\end{aligned}
$$

for $k>0$ and

$$
a_{0}^{(p)}=\frac{e_{0}-1}{-a_{0}}=\frac{\sum_{j=1}^{\left\lfloor\frac{p}{2}\right\rfloor} a_{0 j} c_{k}(j, j)-1}{\sum_{i=1}^{\left\lfloor\frac{p}{2}\right\rfloor} a_{0 j}-a_{00}} .
$$


Theorem 5.5. For $p$ an integer greater than two, let

$$
\begin{aligned}
& f(p, n, m) \\
& =\sum_{|w|=m}\left(\nu_{h}\left(F_{w 0} K\right)+\nu_{h}\left(F_{w 1} K\right)+\nu_{h}\left(F_{w 2} K\right)\right)^{p-3 n}\left(\nu_{h}\left(F_{w 0} K\right) \nu_{h}\left(F_{w 1} K\right) \nu_{h}\left(F_{w 2} K\right)\right)^{n} .
\end{aligned}
$$

For all $p>2$, there is a matrix $M_{p}$ of size $\left\lfloor\frac{p}{3}\right\rfloor+1$ such that

$$
f(p, n, m+1)=\sum_{i=0}^{\left\lfloor\frac{p}{3}\right\rfloor} M_{p}(n+1, i+1) f(p, i, m) .
$$

Proof. It suffices to show that this is true for the case of $m=1$, since we can use this matrix on the subcells of each first level cell and so forth. Since each $\nu_{h}\left(F_{i j} K\right)$ can be written as linear combinations of the first level measures $x, y$, and $z$ using the matrices $E_{i}$, we can expand $f(p, n, 2)$ into a polynomial in terms of the first level measures. This polynomial will be symmetric by the symmetry of $S G$, and therefore can be written in the form

$$
\left.\begin{array}{r}
\sum_{i=0}^{\left\lfloor\frac{p}{3}\right\rfloor}(x y z)^{i}\left[\sum _ { j = 1 } ^ { \lfloor \frac { p - 3 i } { 2 } \rfloor } a _ { i j } \left(x^{j} y^{p-3 i-j}+x^{p-3 i-j} y^{j}+\cdots\right.\right. \\
\left.+y^{j} z^{p-3 i-j}+y^{p-3 i-j} z^{j}\right)+a_{i 0} s(p-3 i)
\end{array}\right]
$$

where $\left(a_{i j}\right)$ are the coefficients. From here, we proceed exactly as in the previous lemma, except that we do not pull out the $i=0$ term (i.e. the sum outside the square brackets) from the sum since we know that $x^{p}+y^{p}+z^{p}$ can be represented by coefficients $\left(a_{k}^{(p)}\right)$ (hence we already know that $\left.M_{p}(1, i+1)=a_{i}^{(p)}\right)$. Hence, we find that

$$
f(p, n, 2)=\sum_{k=0}^{\left\lfloor\frac{p}{3}\right\rfloor} f_{k}^{(n)} \alpha^{p-3 k} \beta^{k}=\sum_{k=0}^{\left\lfloor\frac{p}{3}\right\rfloor} f_{k}^{(n)} f(p, k, 1)
$$

where

$$
f_{k}^{(n)}=\sum_{i=0}^{k} d_{i, k-i}=\sum_{i=0}^{k}\left[\sum_{j=1}^{\left\lfloor\frac{p-3 i}{2}\right\rfloor} a_{i j} c_{k-i}(j, 3 i+j)+\left(a_{i 0}-\sum_{j=1}^{\left\lfloor\frac{p-3 i}{2}\right\rfloor} a_{i j}\right) a_{k-i}^{(p-3 i)}\right] .
$$

Hence, $M_{p}(n+1, k+1)=f_{k}^{(n)}$.

Supposing we have such a matrix $M_{p}$,

$$
P=\left(\nu_{h}(K)^{p-3 j}\left(\nu_{h}\left(F_{0} K\right) \nu_{h}\left(F_{1} K\right) \nu_{h}\left(F_{2} K\right)\right)^{j}\right)_{j=0}^{\left\lfloor\frac{p}{3}\right\rfloor},
$$

and $e_{1}$ is the first standard basis vector, then

$$
\operatorname{dim}_{p} \nu_{h}=\lim _{m \rightarrow \infty} \frac{\log \left(P M_{p}^{m} e_{1}\right)}{m(p-1) \log \frac{3}{5}} .
$$


If one can show that the entries in $M_{p}$ are positive for all integer values $p$ greater than 2 (as seen in the case of $p=3$ and 4 ), then, by the Perron-Frobenius theorem, the maximum eigenvalue will be positive and its associated eigenvector will have all positive entries and we can write

$$
\operatorname{dim}_{p} \nu_{h}=\frac{\log \max \sigma\left(M_{p}\right)}{(p-1) \log \frac{3}{5}}
$$

where $\sigma\left(M_{p}\right)$ is the spectrum of $M_{p}$. However, it is not yet evident how to show this positivity.

Although these matrices are difficult to compute, we can (rather easily) get bounds on $\operatorname{dim}_{p} \nu_{h}$ for $p>1$ (where $p$ no longer needs to be an integer) and can in fact pinpoint the limit as $p$ approaches infinity, which is a corollary from Theorem 4.3 .

Corollary 5.6. Let $\nu_{h}$ be an energy measure and $p>1$ be a real number. Then

$$
\frac{p}{p-1}-\frac{\log 3}{(p-1) \log \frac{5}{3}} \leq \operatorname{dim}_{p} \nu_{h} \leq \frac{p}{p-1} \text {. }
$$

Proof.

$$
\operatorname{dim}_{p} \nu_{h} \leq \lim _{m \rightarrow \infty} \frac{\log \nu_{h}\left(F_{0}^{m} K\right)^{p}}{(p-1) m \log \frac{3}{5}}=\lim _{m \rightarrow \infty} \frac{p \log \nu_{h}\left(F_{0}^{m} K\right)}{(p-1) m \log \frac{3}{5}} \leq \frac{p}{p-1}
$$

since the limit of the log over $m$ converges to the log of the maximum eigenvalue of $E_{0}$. Without loss of generality, assume $\max _{i=0,1,2} \nu_{h}\left(F_{i} K\right)=\nu_{h}\left(F_{0} K\right)$. By Theorem 4.3, we know that

$$
\operatorname{dim}_{p} \nu_{h} \geq \lim _{m \rightarrow \infty} \frac{\log 3^{m} \nu_{h}\left(F_{0}^{m} K\right)^{p}}{(p-1) m \log \frac{3}{5}}=\frac{p}{p-1}-\frac{\log 3}{(p-1) \log \frac{5}{3}} .
$$

It follows that $\lim _{p \rightarrow \infty} \operatorname{dim}_{p} \nu_{h}=1$.

\section{Extensions to $S G_{3}$ and the tetrahedral Sierpinski Gasket}

In this section, we attempt to generalize some of the results in the previous two sections to two other p.c.f. fractals. Specifically, we determine the analogous matrices for the level-three Sierpinksi Gasket, $S G_{3}$, compute the $L_{2}$ dimension of the corresponding energy measure, and define matrices for the Tetrahedral Sierpinski Gasket.

A necessary item to derive for each case is the renormalization constant $r$, such that, as in equation (1.3), the sum of the squares of the differences of a harmonic function on an $m$ th level graph, when multiplied by $r^{-m}$, is the same for all $m$. In equation (1.3) (i.e. for the case of $S G)$ ), $r=\frac{3}{5}$.

The fractal $K=S G_{3}$ is like $S G$, except that there are six contractions instead of three, each of the contractions $F_{0}, \ldots, F_{5}$ mapping to one of the cells as in the above figure. The harmonic extension matrices for $S G_{3}, A_{i}$, which map the boundary values of a harmonic function to the boundary values of the $i$ th cell are

$$
A_{0}=\left[\begin{array}{ccc}
1 & 0 & 0 \\
\frac{8}{15} & \frac{4}{15} & \frac{3}{15} \\
\frac{8}{15} & \frac{3}{15} & \frac{4}{15}
\end{array}\right], \quad A_{3}=\left[\begin{array}{ccc}
\frac{1}{3} & \frac{1}{3} & \frac{1}{3} \\
\frac{3}{15} & \frac{8}{15} & \frac{4}{15} \\
\frac{3}{15} & \frac{4}{15} & \frac{8}{15}
\end{array}\right]
$$




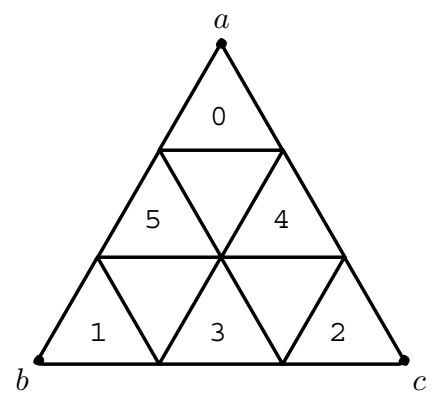

FiguRE 12. First level cells of $S G_{3}$

and $A_{1}$ and $A_{2}$ are generated by cyclic permutations of the columns and rows of $A_{0}$, while $A_{4}$ and $A_{5}$ are generated from $A_{3}$ similarly. We again define the energy measure to be $\nu_{h}\left(F_{w} K\right)=r^{-|w|} \mathcal{E}\left(h \circ F_{w}\right)$. The renormalization constant is $r=\frac{7}{15}$. Proceeding as in section 4 , we first assume one of the boundary values of our harmonic function $h$ to be zero, but for simplicity, we assume the other two are $\sqrt{105} a$ and $\sqrt{105} b$. We then get the following set of equations:

$$
\begin{aligned}
& \nu_{h}\left(F_{0} K\right)=26 a^{2}+26 b^{2}+46 a b, \\
& \nu_{h}\left(F_{1} K\right)=98 a^{2}+26 b^{2}-98 a b, \\
& \nu_{h}\left(F_{2} K\right)=26 a^{2}+98 b^{2}-98 a b, \\
& \nu_{h}\left(F_{3} K\right)=26 a^{2}+26 b^{2}-44 a b, \\
& \nu_{h}\left(F_{4} K\right)=8 a^{2}+26 b^{2}-8 a b, \\
& \nu_{h}\left(F_{5} K\right)=26 a^{2}+8 b^{2}-8 a b .
\end{aligned}
$$

From these polynomials, it is immediate that

$$
\sum_{j=3}^{5} \nu_{h}\left(F_{0} K\right)=\frac{2}{5} \sum_{j=0}^{2} \nu_{h}\left(F_{0} K\right)
$$

Also, the first and last three polynomials form two linearly independent sets. In fact,

$$
\left(\begin{array}{c}
\nu_{h}\left(F_{3} K\right) \\
\nu_{h}\left(F_{4} K\right) \\
\nu_{h}\left(F_{5} K\right)
\end{array}\right)=\frac{1}{60}\left[\begin{array}{ccc}
-2 & 13 & 13 \\
13 & -2 & 13 \\
13 & 13 & -2
\end{array}\right]\left(\begin{array}{c}
\nu_{h}\left(F_{0} K\right) \\
\nu_{h}\left(F_{1} K\right) \\
\nu_{h}\left(F_{2} K\right)
\end{array}\right)
$$

So it suffices to just look at how the "corner measures" are distributed. Proceeding as before, finding polynomial expressions for $\nu_{h}\left(F_{00} K\right), \nu_{h}\left(F_{01} K\right)$, and $\nu_{h}\left(F_{02} K\right)$ and doing some linear algebra on the coefficients, we get that

$$
\left(\begin{array}{c}
\nu_{h}\left(F_{00} K\right) \\
\nu_{h}\left(F_{01} K\right) \\
\nu_{h}\left(F_{02} K\right)
\end{array}\right)=\frac{98}{5^{4} 3^{3}}\left[\begin{array}{ccc}
3701 & -49 & -49 \\
962 & 287 & -238 \\
962 & -238 & 287
\end{array}\right]\left(\begin{array}{c}
\nu_{h}\left(F_{0} K\right) \\
\nu_{h}\left(F_{1} K\right) \\
\nu_{h}\left(F_{2} K\right)
\end{array}\right) .
$$

We let $E_{0}$ equal this matrix, and $E_{1}$ and $E_{2}$ can be derived using the symmetry of $S G_{3}$ as we used the symmetry of $S G$ in section 4 . Similarly, we can show that

$$
\left(\begin{array}{c}
\nu_{h}\left(F_{30} K\right) \\
\nu_{h}\left(F_{31} K\right) \\
\nu_{h}\left(F_{32} K\right)
\end{array}\right)=\frac{1}{5^{4} 3^{3} 4}\left[\begin{array}{ccc}
1174 & 49 & 49 \\
-962 & 3613 & 1213 \\
962 & 1213 & 3613
\end{array}\right]\left(\begin{array}{c}
\nu_{h}\left(F_{0} K\right) \\
\nu_{h}\left(F_{1} K\right) \\
\nu_{h}\left(F_{2} K\right)
\end{array}\right)
$$


We let $E_{3}$ be this matrix and derive $E_{4}$ and $E_{5}$ using symmetry. Let $C$ be the matrix in (6.2) and let $e=\left(\nu_{h}\left(F_{0} K\right), \nu_{h}\left(F_{1} K\right), \nu_{h}\left(F_{2} K\right)\right)^{T}$. Then

$$
\nu_{h}\left(F_{w} K\right)=r^{-|w|} \mathcal{E}\left(h \circ F_{w}\right)=\left(\begin{array}{lll}
1 & 1 & 1
\end{array}\right)(I+C) E_{w_{m}} \cdots E_{w_{1}} e
$$

where $I$ is the $3 \times 3$ identity matrix.

Let $x, y, z$ denote the measures of $F_{0} K, F_{1} K$, and $F_{2} K$ respectively, and $x^{\prime}, y^{\prime}, z^{\prime}$ denote the other three measures respectively. Solving for $a^{2}, b^{2}$, and $a b$ and proceeding as in Theorem 4.2 we get the following relations:

and

$$
x y+x z+y z=\frac{481}{913}\left(x^{2}+y^{2}+z^{2}\right)
$$

It follows that

$$
x^{\prime} y^{\prime}+x^{\prime} z^{\prime}+y^{\prime} z^{\prime}=\frac{91}{118}\left(x^{\prime 2}+y^{\prime 2}+z^{\prime 2}\right) .
$$

and

$$
x^{2}+y^{2}+z^{2}=\frac{913}{1875}(x+y+z)^{2}
$$

$x^{\prime 2}+y^{\prime 2}+z^{\prime 2}=\frac{59}{150}\left(x^{\prime}+y^{\prime}+z^{\prime}\right)^{2}=\frac{59}{150}\left(<\frac{2}{5}\right)^{2}(x+y+z)=\frac{118}{1875}(x+y+z)$.

So

$x^{2}+y^{2}+z^{2}+x^{\prime 2}+y^{\prime 2}+z^{\prime 2}=\frac{1031}{1875}(x+y+z)=\frac{1031}{1875} \frac{5}{7}\left(x+y+z+x^{\prime}+y^{\prime}+z^{\prime}\right)$.

Therefore, since the same relation holds for the energy measures, we get

$$
\sum_{i=0}^{5} \nu_{h}\left(F_{i} K\right)^{2}=\frac{1031}{2625} \nu_{h}(K)
$$

Hence, we get the following theorem:

Theorem 6.1. Let $\nu_{h}$ be an energy measure on $S G_{3}$. Then

$$
\operatorname{dim}_{2} \nu_{h}=\frac{\log \frac{2625}{1031}}{\log \frac{15}{7}}
$$

We can look at the measure of a cell of a fractal as an $n-1$ variable, $\left(\begin{array}{l}n \\ 2\end{array}\right)$-term polynomial (or vector) where $n$ is the number of boundary points of the fractal. The significance of $S G_{3}$ is that the number of first level cells exceeds the maximal number of linearly independent cell measures needed to span all polynomials. In that case, we see that we need only look at the "corner subcells" of each cell, since those fix the other three "middle cells" with the matrix $C$. The tetrahedral $S G$, or $S G^{4}$, addresses the case of when the number of first level cells is less than the number of polynomials necessary to make a basis. In this case, we have four first level cells, but the measure polynomials have six terms, so they don't form a basis for the six-dimensional space of polynomials and will not necessarily span the rest of the cell measures. In fact, they don't.

However, we can still define matrices for a linear extension algorithm for the energy measures on the $S G^{4}$ by taking the second level cells as our first level, in which case we have sixteen total cells, more than enough to make a linearly independent set. In general, if a fractal does not have enough first level cells to 

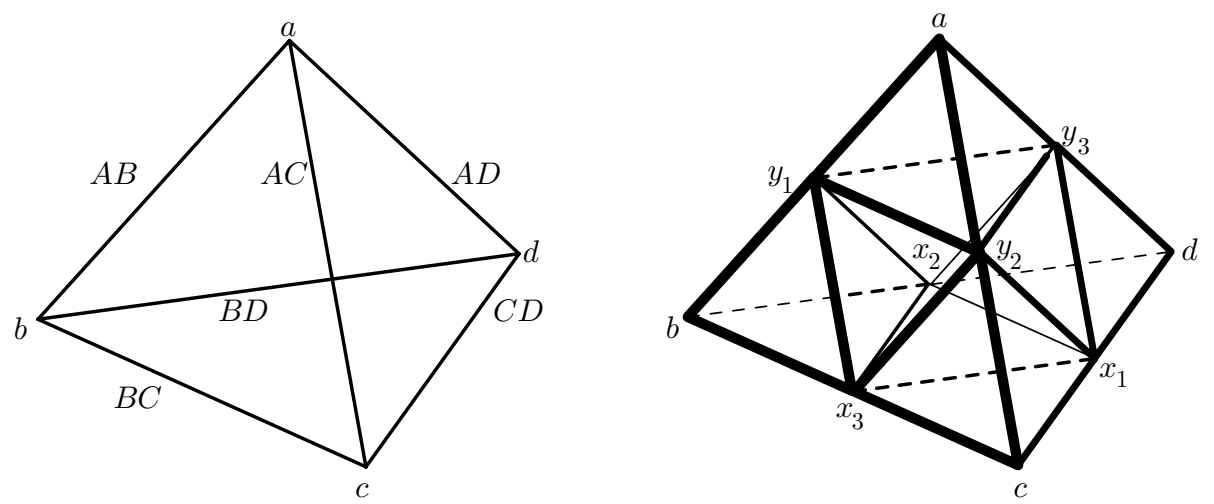

Figure 13. First and second level graphs of $S G^{4}$

make an independent set, then if the cells in the next level are spanned by those in the first level, then all cells are spanned by the first level cells and we're done; if no, then choose a new independent set by adding the cell that is not in the span and, recursively, we can construct a linearly independent set of cell measures. As we shall see, we need only go to level two for $S G^{4}$.

$S G^{4}$ is the fractal $K$ satisfying $\bigcup_{i=0}^{3} F_{i}(K)=K$, where $F_{i} x=\frac{1}{2} x+q_{i}$ and $q_{0}=(1 / 2,1 / 2,1), q_{1}=(0,0,0), q_{2}=(1,0,0), q_{3}=(1 / 2,1,0)$. So, as in the above figure, $F_{0}$ contracts $K$ to the tetrahedron containing $a, F_{0}$ to the one containing $b$, et cetera. As with $S G$, we approximate $S G^{4}$ by a sequence of graphs $\Gamma_{m}$ with vertices $V_{m}$, defining edge relations similarly (see the introduction).

We need to determine the harmonic extension matrices that extend the values on $V_{0}$ of a harmonic function to the vertices of $V_{1}$ (as displayed in Figure 13). To do this, we fix the four boundary values of $S G^{4}$ to be $a, b, c$, and $d$ (and label those points as $A, B$, etc.). We let the other six points on the first level graph of $S G^{4}$ be $x_{1}, x_{2}, x_{3}, y_{1}, y_{2}, y_{3}$. Computing the energy and setting the partial derivatives with respect to each variable equal to zero, we get the following linear equations:

$$
\begin{array}{ll}
6 x_{1}-x_{2}-x_{3}-y_{2}-y_{3} & =c+d \\
-x_{1}+6 x_{2}-x_{3}-y_{1}-y_{3} & =b+d \\
-x_{1}-x_{2}+6 x_{3}-y_{1}-y_{2} & =b+c \\
-x_{2}-x_{3}+6 y_{1}-y_{2}-y_{3} & =a+b \\
-x_{1}-x_{3}-y_{1}+6 y_{2}-y_{3} & =a+c \\
-x_{1}-x_{2}-y_{1}-y_{2}+6 y_{3} & =a+d .
\end{array}
$$

After some linear algebra, we find that

$$
\begin{aligned}
& x_{1}=\frac{1}{6} a+\frac{1}{6} b+\frac{1}{3} c+\frac{1}{3} d, \\
& x_{2}=\frac{1}{6} a+\frac{1}{3} b+\frac{1}{6} c+\frac{1}{3} d, \\
& x_{3}=\frac{1}{6} a+\frac{1}{3} b+\frac{1}{3} c+\frac{1}{6} d, \\
& y_{1}=\frac{1}{3} a+\frac{1}{3} b+\frac{1}{6} c+\frac{1}{6} d, \\
& y_{2}=\frac{1}{3} a+\frac{1}{6} b+\frac{1}{3} c+\frac{1}{6} d, \\
& y_{3}=\frac{1}{3} a+\frac{1}{6} b+\frac{1}{6} c+\frac{1}{3} d .
\end{aligned}
$$


Hence,

$$
A_{0}=\left[\begin{array}{llll}
1 & 0 & 0 & 0 \\
\frac{1}{3} & \frac{1}{3} & \frac{1}{6} & \frac{1}{6} \\
\frac{1}{3} & \frac{1}{6} & \frac{1}{3} & \frac{1}{6} \\
\frac{1}{3} & \frac{1}{6} & \frac{1}{6} & \frac{1}{3}
\end{array}\right]
$$

and $A_{1}, A_{2}$, and $A_{3}$ are defined similarly.

If we compute the second level energy on $S G^{4}$ and compare it to the first level energy, we find that the renormalization constant is $r=\frac{2}{3}$.

We define $\nu_{h}$ as in the previous cases and let $h$ be the harmonic function with boundary values $0, a, b, c$. Then the polynomials for the measures of $F_{01} K, F_{02} K$, $F_{03} K, F_{11} K, F_{22} K$, and $F_{33} K$ form a linearly independent set, where

$$
\begin{aligned}
& \nu_{h}\left(F_{01} K\right)=\frac{1}{72}\left(11 a^{2}+b^{2}+c^{2}+a b+a c+b c\right), \\
& \nu_{h}\left(F_{02} K\right)=\frac{1}{72}\left(a^{2}+11 b^{2}+c^{2}+a b+a c+b c\right), \\
& \nu_{h}\left(F_{03} K\right)=\frac{1}{72}\left(a^{2}+b^{2}+11 c^{2}+a b+a c+b c\right), \\
& \nu_{h}\left(F_{11} K\right)=\frac{1}{72}\left(96 a^{2}+11 b^{2}+11 c^{2}-64 a b-64 a c+21 b c\right), \\
& \nu_{h}\left(F_{22} K\right)=\frac{1}{72}\left(11 a^{2}+96 b^{2}+11 c^{2}-64 a b+21 a c-64 b c\right), \\
& \nu_{h}\left(F_{33} K\right)=\frac{1}{72}\left(11 a^{2}+11 b^{2}+96 c^{2}+21 a b-64 a c-64 b c\right) .
\end{aligned}
$$

Also,

$$
\begin{aligned}
& \nu_{h}\left(F_{001} K\right)=\frac{1}{1728}\left(51 a^{2}+21 b^{2}+21 c^{2}+61 a b+61 a c+41 b c\right), \\
& \nu_{h}\left(F_{002} K\right)=\frac{1}{1728}\left(21 a^{2}+51 b^{2}+21 c^{2}+61 a b+41 a c+61 b c\right), \\
& \nu_{h}\left(F_{003} K\right)=\frac{1}{1728}\left(21 a^{2}+21 b^{2}+51 c^{2}+41 a b+61 a c+61 b c\right), \\
& \nu_{h}\left(F_{011} K\right)=\frac{1}{1728}\left(171 a^{2}+b^{2}+c^{2}+a b+a c+b c\right), \\
& \nu_{h}\left(F_{022} K\right)=\frac{1}{1728}\left(a^{2}+171 b^{2}+c^{2}+a b+a c+b c\right), \\
& \nu_{h}\left(F_{033} K\right)=\frac{1}{1728}\left(a^{2}+b^{2}+171 c^{2}+a b+a c+b c\right) .
\end{aligned}
$$

The matrix relating the first set of linear equations to the second is

$$
E_{0}=\frac{1}{142392}\left[\begin{array}{cccccc}
52853 & 23188 & 23188 & -3016 & -1620 & -1620 \\
23188 & 52853 & 23188 & -1620 & -3016 & -1620 \\
23188 & 23188 & 52853 & -1620 & -1620 & -3016 \\
88621 & -12240 & -12240 & 544 & 544 & 544 \\
-12240 & 88621 & -12240 & 544 & 544 & 544 \\
-12240 & -12240 & 88621 & 544 & 544 & 544
\end{array}\right]
$$

Unlike the previous cases, however, we cannot obtain the other matrices by cyclic permutations because we lose symmetry. Nonetheless, we can still solve for the other matrices by computing the measures for $\nu_{h}\left(F_{101} K\right), \nu_{h}\left(F_{102} K\right)$, and so on, and solving for the matrix that relates the set of measures in (6.4) to those in $F_{1} K$, and we get

$$
E_{1}=\frac{1}{142392}\left[\begin{array}{cccccc}
28084 & -7514 & -7514 & 7725 & 396 & 396 \\
4437 & 4437 & -1496 & 431 & 82 & 82 \\
4437 & -1496 & 4437 & 431 & 82 & 82 \\
-544 & -544 & -544 & 95053 & -224 & -224 \\
-37825 & -37825 & 63036 & 14439 & 14090 & -9642 \\
-37825 & 63036 & -37825 & 14439 & -9642 & 14090
\end{array}\right]
$$


and similarly, by the rotational symmetry of our choice of cells,

$$
E_{2}=\frac{1}{142392}\left[\begin{array}{cccccc}
4437 & 4437 & -1496 & 82 & 431 & 82 \\
-7514 & 28084 & -7514 & 396 & 7725 & 396 \\
-1496 & 4437 & 4437 & 82 & 431 & 82 \\
-37825 & -37825 & 63036 & 14090 & 14439 & -9642 \\
-544 & -544 & -544 & -224 & 95053 & -224 \\
63036 & -37825 & -37825 & -9642 & 14439 & 14090
\end{array}\right]
$$

and

$$
E_{3}=\frac{1}{142392}\left[\begin{array}{cccccc}
4437 & -1496 & 4437 & 82 & 82 & 431 \\
-1496 & 4437 & 4437 & 82 & 82 & 431 \\
-7514 & -7514 & 28084 & 396 & 396 & 7725 \\
-37825 & 63036 & -37825 & 14090 & -9642 & 14439 \\
63036 & -37825 & -37825 & -9642 & 14090 & 14439 \\
-544 & -544 & -544 & -224 & -224 & 95053
\end{array}\right]
$$

Now, we can relate $\nu_{h}\left(F_{01} K\right), \ldots, \nu_{h}\left(F_{33}\right)$ to the measures of the other cells,

$$
\begin{aligned}
& \nu_{h}\left(F_{00} K\right)=\frac{1}{72}\left(11 a^{2}+11 b^{2}+11 c^{2}+21 a b+21 a c+21 b c\right), \\
& \nu_{h}\left(F_{10} K\right)=\frac{1}{72}\left(16 a^{2}+b^{2}+c^{2}-4 a b-4 a c+b c\right), \\
& \nu_{h}\left(F_{12} K\right)=\frac{1}{72}\left(16 a^{2}+11 b^{2}+c^{2}-24 a b-4 a c+b c\right), \\
& \nu_{h}\left(F_{13} K\right)=\frac{1}{72}\left(16 a^{2}+b^{2}+11 c^{2}-4 a b-24 a c+b c\right), \\
& \nu_{h}\left(F_{20} K\right)=\frac{1}{72}\left(a^{2}+16 b^{2}+c^{2}-4 a b+a c-4 b c\right), \\
& \nu_{h}\left(F_{21} K\right)=\frac{1}{72}\left(11 a^{2}+16 b^{2}+c^{2}-24 a b+a c-4 b c\right), \\
& \nu_{h}\left(F_{23} K\right)=\frac{1}{72}\left(a^{2}+16 b^{2}+11 c^{2}-4 a b+a c-24 b c\right), \\
& \nu_{h}\left(F_{30} K\right)=\frac{1}{72}\left(a^{2}+b^{2}+16 c^{2}+a b-4 a c-4 b c\right), \\
& \nu_{h}\left(F_{31} K\right)=\frac{1}{72}\left(11 a^{2}+b^{2}+16 c^{2}+a b-24 a c-4 b c\right), \\
& \nu_{h}\left(F_{32} K\right)=\frac{1}{72}\left(a^{2}+11 b^{2}+16 c^{2}+a b-4 a c-24 b c\right),
\end{aligned}
$$

by the matrix

$$
M=\frac{1}{5933}\left[\begin{array}{cccccc}
12427 & 12427 & 12427 & -816 & -816 & -816 \\
5202 & -731 & -731 & 397 & 48 & 48 \\
-2193 & -2193 & 3740 & 1191 & 842 & -554 \\
-2193 & 3740 & -2193 & 1191 & -554 & 842 \\
-731 & 5202 & -731 & 48 & 397 & 48 \\
-2193 & -2193 & 3740 & 842 & 1191 & -554 \\
3740 & -2193 & -2193 & -554 & 1191 & 842 \\
-731 & -731 & 5202 & 48 & 48 & 397 \\
-2193 & 3740 & -2193 & 842 & -554 & 1191 \\
3740 & -2193 & -2193 & -554 & 842 & 1191
\end{array}\right] .
$$


Therefore, for $e=\left(\nu_{h}\left(F_{00} K\right), \nu_{h}\left(F_{01} K\right), \ldots, \nu_{h}\left(F_{33} K\right)\right)^{T}$, we have

$$
\nu_{h}\left(F_{w} K\right)=\left(\begin{array}{llllll}
1 & 1 & 1 & 1 & 1 & 1
\end{array}\right)(I+M) E_{w_{m}} \cdots E_{w_{1}} e .
$$

\section{ACKNOWLEDGEMENTS}

We are grateful to Martin Kassabov and Jun Kigami for helpful discussions.

\section{REFERENCES}

1. M. T. Barlow, R. F. Bass, and T. Kumagai, Stability of parabolic Harnack inequalities on metric measure spaces, J. Math. Soc. Japan 58 (2006), 485-519. MR2228569 (2007f:60064)

2. O. Ben-Bassat, R. S. Strichartz, and A. Teplyaev, What is not in the domain of the Laplacian on a Sierpinski gasket type fractal, J. Funct. Anal. 166 (1999), 197-217. MR1707752 (2001e:31016)

3. B. Bockelman and R. S. Strichartz, Partial differential equations on products of Sierpinski gaskets.

4. M. Fukushima and T. Shima, On a spectral analysis for the Sierpinski gasket, Potential Anal. 1 (1992), 1-35. MR1245223 (95b:31009)

5. M. Hino, On singularity of energy measures on self-similar sets, Probab. Theory Related Fields 132 (2005), 265-290. MR2199293

6. M. Hino and K. Nakahara, On singularity of energy measures on self-similar sets II, Bull. London Math. Soc. 38 (2006), 1019-1032. MR2285256

7. J. Kigami, Harmonic metric and Dirichlet form on the Sierpinski gasket, Asymptotic problems in probability theory: stochastic models and diffusions on fractals (Sanda/Kyoto 1990) (K. D. Elworthy and N. Ikeda, eds.), 283, Longman Sci. Tech., 1993, pp. 201-218. MR1354156 (96m:31014)

8. _ Analysis on fractals, Cambridge University Press, New York, 2001. MR1840042 (2002c:28015)

9. Harmonic analysis for resistance forms, J. Funct. Anal. 204 (2003), 399-444. MR2017320 (2004m:31010)

10. J. Kigami and M. L. Lapidus, Weyl's problem for the spectral distribution of Laplacians on p.c.f. self-similar fractals, Comm. Math. Phys. 158 (1993), 93-125. MR1243717 (94m:58225)

11. S. Kusuoka, Dirichlet forms on fractals and products of random matrices, Publ. Res. Inst. Math. Sci. 25 (1989), 659-680. MR1025071 (91m:60142)

12. Lecture on diffusion process on nested fractals, Lecture Notes in Math. 1567 (1993).

13. R. Myers, R. S. Strichartz, and A. Taplyaev, Dirichlet forms on the Sierpinski gasket, Pacific J. Math 217 (2004), 149-174. MR2105771 (2005k:31028)

14. K. A. Okoudjou and R. S. Strichartz, Asymptotics of eigenvalue clusters for Schrödinger operators on the Sierpinski gasket, Preprint.

15. T. Shima, On eigenvalue problems for the random walks on the Sierpinski pre-gaskets, Japan J. Indust. Appl. Math. 8 (1991), 127-141. MR1093832 (92g:60094)

16. - On eigenvalue problems for Laplacians on p.c.f. self-similar sets, Japan J. Indust. Appl. Math. 13 (1996), 1-23. MR1377456 (97f:28028)

17. R. S. Strichartz, Self-similar measures and their Fourier transforms. III, Indiana Univ. Math. J. 42 (1993), 367-411. MR1237052 (94j:42025)

18. Analysis on fractals, Notices Amer. Math. Soc. 46 (1999), 1199-1208. MR1715511 (2000i:58035)

19. , Function spaces on fractals, J. Funct. Anal. 198 (2003), 48-83. MR1962353 (2003m:46058)

20. _ Analysis on products of fractals, Trans. Amer. Math. Soc. 357 (2005), 571-615. MR2095624 (2005m:31016)

21. L Laplacians on fractals with spectral gaps have nice Fourier series, Math. Research Letters 12 (2005), 269-274. MR2150883 (2006e:28013)

22. Differential equations on fractals: A tutorial, Princeton University Press, Princeton, NJ, 2006. MR2246975 (2007f:35003) 
23. A. Taplyaev, Harmonic coordinates on fractals with finitely ramified cell structure, Preprint.

24. (2001h:31012)

Department of Mathematics, University of Nebraska, Lincoln, Nebraska 68588 Current address: Department of Mathematics, UCLA, Los Angeles, California 90095

E-mail address: jonasazzam@ucla.edu

Department of Mathematics, University of Maryland, College Park, Maryland 20742 Current address: Department of Mathematics, UCLA, Los Angeles, California 90095 E-mail address: michaelhall@ucla.edu

Department of Mathematics, Malott Hall, Cornell University, Ithaca, New York 14853

E-mail address: str@math.cornell.edu 